\title{
On the suboptimality of single-factor exercise strategies for Bermudan swaptions
}

\author{
Mikkel Svenstrup* \\ Department of Finance \\ The Aarhus School of Business \\ e-mail: msv@asb.dk
}

28. Nov. 2002

(first version 6.Aug.2002)

\begin{abstract}
In this paper we examine the cost of using recalibrated single-factor models to determine the exercise strategy for Bermudan swaptions in a multi-factor world. We demonstrate that single-factor exercise strategies applied in a multi-factor world only give rise to economically insignificant losses. Furthermore, we find that the conditional model risk as defined in Longstaff, Santa-Clara \& Schwartz (2001), is statistically insignificant given the number of observations. Additional tests using the Primal-Dual algorithm of Andersen \& Broadie (2001) indicate that losses found in Longstaff et al. (2001) cannot as claimed be ascribed to the number of factors. Finally we find that for valuation of Bermudan swaptions with long exercise periods, the simple approach proposed in Andersen (2000) is outperformed by the Least Square Monte Carlo method of Longstaff \& Schwartz (2001) and, surprisingly, also by the exercise strategies from the single-factor models.
\end{abstract}

JEL classification: C52; E43; E47; G12; G13;

Keywords: Bermudan swaption; American option; Least Square Monte Carlo; Libor Market Model; Model Risk; Model Calibration

\section{Introduction}

The notional amount of the contracts in the OTC market for interest rate derivatives contracts has been growing rapidly to an estimated $\$ 90$ trillion. Of these interest rate swaps is by far the largest single group with outstanding contracts

*The author acknowlege comments and suggestions from Tom Engsted, Svend Jakobsen, Malene S. Jensen, Nicki S. Rasmussen and Søren Willemann. 
of $\$ 68$ trillion followed by interest rate options with $\$ 12.5$ trillion. The total market value is estimated to $\$ 2.5$ trillion $\mathrm{be}^{1}$.

As discussed in e.g. Green \& Figlewski (1999) the public is usually buying options leaving the dealer community with an overall short position. Several sources of risk have been identified in relation to derivatives trading, and in particular model risk ${ }^{2}$ has been accentuated as the nominal amounts and complexity of derivative contracts have increased. As a consequence of this, the vast majority of the literature has been focusing on the choice of interest rate derivative models in relation to the hedging and valuation performance from the option writers view ${ }^{3}$.

Another important aspect of model risk is faced by holders of American style options. By choosing a particular model, holders implicitly define their exercise strategy, but as stressed in Longstaff et al. (2001, p.43) "...in an efficient market, an American option is only worth its market value to an investor who follows the optimal strategy". The expected present value of the cash flow from an American option is therefore less than the market value if anything but the optimal strategy is followed. The quantitative effect of using a slightly suboptimal exercise strategy is likely to differ from asset to asset, but it is important to stress that the quality of an exercise strategy can be measured in terms of the expected discounted cash flow obtained by following it. Better strategies lead to higher expected present values.

In this paper we concentrate on one of the most liquid American style interest rate derivatives, namely Bermudan swaptions. Recent studies by Longstaff et al. (2001) and Andersen \& Andreasen (2001) have come to opposite conclusions about the significance of using slightly suboptimal exercise strategies in the market for Bermudan swaptions, and we therefore take these two papers as a starting point for a closer analysis.

In Longstaff et al. (2001) it is argued that the present value losses from using suboptimal exercise strategies are economically significant for swaption holders even if they are within bid/ask spreads, as they could have been avoided by using better strategies. In particular, they claim that the Wall Street practice of using the exercise strategies from single-factor models continuously recalibrated to market information, costs the holders of Bermudan swaptions billions of dollars as a whole. Furthermore, they argue that the present value cost conditional on making the wrong exercise decision is substantial and constitutes a new dimension to the potential effects of model risk. These conclusions are based on an extensive simulation experiment designed to replicate the Wall Street practice.

Contrary to Longstaff et al. (2001), the study by Andersen \& Andreasen (2001) argues that using exercise strategies derived from a best fit single-factor

\footnotetext{
${ }^{1}$ The estimates are based on the November 2002 press release from the Bank for International Settlements.

${ }^{2}$ See also Rebonato (n.d.a) for a good discussion of model risk.

${ }^{3}$ See e.g. Bühler, Uhrig-Homburg, Walter \& Weber (1999), Green \& Figlewski (1999), Hull \& Suo (2001), Collin-Dufresne \& Goldstein (2002), Driessen, Klaassen \& Melenberg (2000), Gupta \& Subrahmanyam (2002), Rebonato (n.d.a) etc.
} 
model only results in insignificant losses and provides a good mark-to-market of the Bermudan prices. Furthermore, they find that single-factor models, when calibrated appropriately to the prices of caps and European swaptions from a two-factor model, give Bermudan swaption prices that are slightly higher than those from the two-factor models.

Both these papers use simulation based valuation techniques in order to estimate the lower bounds for the true value of the Bermudan swaptions. However, they apply two different approaches for estimating the optimal exercise strategy. Longstaff et al. (2001) use the Least Square Monte Carlo technique by Longstaff \& Schwartz (2001) (LSM), while Andersen \& Andreasen (2001) apply the "simple approach" by Andersen (2000) (LAM).

In this paper we merge the approaches taken in these papers into a unified framework in order to facilitate a direct comparison. As the benchmark multifactor model we set up a four-factor log-normal Libor market model based on a principal component analysis of the Euro forward-rate curve.

We start out by estimating the true Bermudan swaption values in this model using both the LAM and LSM exercise strategies. Interestingly, we find that the LAM strategy is outperformed by the LSM strategy, in particular when the exercise period is long. Furthermore, using the Primal-Dual algorithm by Andersen \& Broadie (2001), we are able to conclude that the LSM strategies are in fact very close to being optimal.

Having established benchmark model prices for the Bermudan swaptions, we set up three different single-factor interest rate models including one spot-rate model (Black, Derman \& Toy (1990)) and two forward-rate models within the Heath, Jarrow \& Morton (1992) class (Ritchken \& Sankarasubramanian (1995), Andreasen (2000)).

Using these we construct a total of six different single-factor exercise strategies and use these to compute lower bounds on a set of Bermudan swaptions. The application of efficiency improvement techniques allows us to give reasonably precise estimates. For three of these single-factor exercise strategies the lower bounds are virtually identical to the LSM values, leading us to conclude that losses from following exercise strategies from recalibrated single-factor models in the Bermudan swaptions market, are insignificant and economically irrelevant. This corresponds closely to the findings by Andersen \& Andreasen (2001), but we stress that we have controlled for the optimality of the LSM strategy.

Turning to the conditional present value losses documented by Longstaff et al. (2001), we repeat the exact same simulation procedure but using our singlefactor models. The best performing single-factor models perform reasonably well and the corresponding conditional losses relative to the LSM strategy are both negative and positive. More importantly, the losses are not significantly different from zero when using the same number of paths as in Longstaff et al. (2001). No standard errors on the loss estimates have been reported in Longstaff et al. (2001), but we find crude Monte Carlo estimation to be too inaccurate to conclude anything due to the sizes of the standard errors. The conditional losses are not examined by Andersen \& Andreasen (2001).

We therefore test one single-factor exercise strategy in the Dual-Primal al- 
gorithm of Andersen \& Broadie (2001), which is extremely slow due to the combination of nested simulations and computationally costly exercise decisions. In particular, we find that the upper bounds for the potential losses are very small and often lower than those from the full LSM approach.

This paper is organized as follows. First, we fix some notation regarding Bermudan swaptions. Secondly, we go through the simulation methodology and the benchmark multi-factor model. Then the exercise strategies are described in more detail and the ways to assess the true Bermudan prices are discussed. This is followed by a short outline of the single-factor models as well as their numerical implementation and calibration. All this lead us to the numerical results and finally we make our conclusions.

\section{Notation and definitions}

A forward swap is a standard financial contract where two parties agree to exchange a fixed coupon for a floating rate over a period of time. When paying/receiving fixed for floating this is termed a Payer/Receiver swap. Let $t_{s}$ and $t_{e}$ denote the forward starting date and the final maturity of the swap. Usually the first payment in the swap is fixed on $t_{s}$ and paid on $t_{s+1}$ and the last payment is fixed at time $t_{e-1}$ and paid at time $t_{e}$. If we let $P(t, T)$ denote the time $t$ value of a discount bond with maturity $T$, then the time $t$ value of a payer swap with coupon $\theta$ is

$$
S_{s, e}(t)=P\left(t, t_{s}\right)-P\left(t, t_{e}\right)-\theta \sum_{i=s}^{e-1} P\left(t, t_{i+1}\right)\left(t_{i+1}-t_{i}\right), t \leq t_{s} .
$$

A standard payer Bermudan swaption $B S_{s, e}$ gives the holder the right, but not the obligation, to enter into the forward payer swap with a final maturity $t_{e}$ and a coupon $\theta$, on a set of times $t_{s}, \ldots, t_{e-1}$. As $t$ exceeds tenor times we adjust the formula by only summing remaining payments. For this purpose we define $n(t)$ to be the mapping from a time into the swap's next reset time after $t$, i.e. $t_{n(t)}<t \leq t_{n(t)}$

$$
S_{s, e}(t)=P\left(t, t_{n(t)}\right)-P\left(t, t_{e}\right)-\theta \sum_{i=n(t)}^{e-1} P\left(t, t_{i+1}\right)\left(t_{i+1}-t_{i}\right), t \leq t_{e} .
$$

That is, the intrinsic value $X_{t}$ at an exercise time is $\max \left(S_{n(t), e}(t), 0\right)$. This intrinsic value is exactly the value of the European swaption $E S_{n(t), e}(t)$ of the swap. In this paper we define the Bermudan premium as the difference between the Bermudan swaption and the first to mature European swaption.

We make the standard assumptions of no arbitrage and complete and frictionless markets defined on a probability space $\left(\Omega,\left\{\mathcal{F}_{t}\right\}_{t \geq 0}, \mathbb{P}\right)$, where $\left\{\mathcal{F}_{t}\right\}$ is a filtration and $\mathbb{P}$ the physical measure. Let $\mathbb{Q}$ denote the pricing measure induced by the numeraire asset $\beta$ with the associated conditional expectation 
$E^{\mathbb{Q}}\left(\cdot \mid \mathcal{F}_{t}\right)$. The problem of pricing Bermudan swaptions is basically an optimal stopping problem,

$$
B S_{s, e}(t)=\sup _{\tau \in \Gamma(t)} E^{\mathbb{Q}}\left(\frac{\beta_{t}}{\beta_{\tau}} X_{\tau} \mid \mathcal{F}_{t}\right),
$$

where $\Gamma(t)$ is the set of all $\mathcal{F}_{t}$-optional stopping times taking values in the set of exercise times from time $t$ and on.

As should be well known (see e.g. Duffie (1996)), American style options should only be exercised when the intrinsic value $X_{t}$ exceeds the continuation value of the option $B S_{s, e}^{+}(t)$, which defines the optimal exercise time

$$
\tau^{*}=\inf _{t_{s} \leq t_{i}<t_{e}}\left(X_{t_{i}} \geq B S_{s, e}^{+}\left(t_{i}\right)\right) .
$$

Bermudan swaptions are in effect the right to choose between different swaps at different points in time. These core swap rates are the forward starting swap rates $S R_{i, e}, i=s, . ., e-1$ with same final maturity $t_{e}$ as the swap in question. According to the literature (e.g. Andreasen (2000), Rebonato (2000)), it is not enough to know the right terminal distributions for the swap rates involved. An important part of pricing Bermudan swaptions is to have the right termcorrelation (see appendix A.2) structure of the core swap rates.

\section{$3 \quad$ Methodology}

In this section we briefly summarize the simulation approach used by Longstaff et al. (2001) to investigate the effect of single-factor exercise strategies in a multifactor world. They emphasize that even if various models have been calibrated to match a set of market prices from a multi-factor world perfectly, their implied exercise strategies will still be suboptimal.

The basic procedure goes like this. First, simulate a path from the multifactor model to the first exercise date of the Bermudan swaption. Compute the yield curve and the European swaption prices from the benchmark model. Calibrate the single-factor model to this "market" information. Now, if exercise is implied by the single-factor model, we receive the discounted payoff from the benchmark model. If not, we advance the simulation to the next exercise date and so on until the Bermudan swaption has either been exercised or has expired. The expected present value loss is the difference between the average discounted payoff received by following a suboptimal exercise strategy and the discounted payoff received by following the optimal exercise strategy.

\section{The benchmark multi-factor model}

We choose as benchmark model a multi-factor log-normal Libor market model defined on a fixed tenor grid of 0.50 years. The Libor market models of Miltersen, Sandmann \& Sondermann (1997), Brace \& Musiela (1997) and Jamshidian (1997) have become increasingly popular among practitioners, in particular 
because they provide closed form solutions for both caps and European swaptions (though not in the same model). The reason for choosing a different benchmark model than Longstaff et al. (2001) is that the string model (see e.g. Santa-Clara \& Sornette (2001)) applied in their analysis does not have closed form solutions for caps and European swaptions. In order to do the calibration of their one-factor models to the string model, they use the prices of ATM European swaptions extracted from an LSM regression. Although asymptotically unbiased, the size of the standard errors of these price estimates are also of importance but they are not reported in their study. In particular these price estimates are input to the calibration of the single-factor models and they could in principle distort the entire calibration. Therefore we use a Libor market model which does not involve such problems due to the existence of accurate approximations for the prices of European swaptions.

In the Libor market model the fundamental state variables are the discretely compounded forward-rates $F_{k}(t), k=0, . ., K$ corresponding to a given tenor structure $t_{0}<t_{0}+\delta<t_{0}+2 \delta<. .<t_{K}$. The dynamics of the forward rates under the spot Libor measure $\mathbb{Q}$ (see Jamshidian (1997)) can be written as

$$
d F_{k}(t)=\mu(t, F(t)) d t+F_{k}(t) \lambda_{k}(t) \cdot d W_{t}^{\mathbb{Q}}, k=1, . ., K,
$$

where $\mu(t, F(t))$ is a function of the entire forward rate curve and constructed to ensure no arbitrage. $W_{t}^{\mathbb{Q}}$ is an n-dimensional Wiener process and $\lambda_{k}(t)$ is a $n$-dimensional vector of the factor loadings of $F_{k}(t)$ on the Wiener process at time $t$. Notice that the dimension of the state variable is $K$, which could easily be as high as 120 , when the last payment time is 30 year and there are quarterly payments.

\subsection{Estimation of the benchmark model}

We set up the benchmark model using the historical covariance matrix $H$ of the percentage changes in the forward rates. This matrix can be written as

$$
H=V^{\top} \Lambda V
$$

where $\Lambda$ is a diagonal matrix of the eigenvalues (which are positive) and $V$ the matrix of eigenvectors. We follow Longstaff et al. (2001) who make the identifying assumption that the implied covariance matrix $\Sigma$ used in the swaptions market is

$$
\Sigma=V^{\top} \Psi V
$$

where $\Psi$ is a diagonal matrix of implied eigenvalues. This implicitly means that the factors that generate the historical covariance matrix also generate the implied covariance matrix, and if desired we could calibrate the model to match market quoted European swaptions on a given day using the diagonal in $\Psi$ as free parameters. However, for the purpose of this study there is no need to match the implied swaptions volatilities on a particular date.

The historical covariance matrix used in this paper is estimated from daily changes in the forward Libor rates. The forward rates are calculated from yield 
curves estimated daily on a sample consisting of 3-month and 1-year EURIBOR and EUR swap rates using a cubic spline method ${ }^{4}$. The sample covers the period from Jan. 4th, 1999 to Oct. 3rd, 2002 which corresponds to 942 trade dates with observations on all 6 month forward Libor rates from 0.5 to 30 year. To reduce the data input we only include forward rates with maturities of $0.5,1$, $1.5, \ldots .5,6,7,10,15,20,25$ and 30 year. It is well known that even if the covariance matrix has full rank, it is common that a relatively low number of factors are needed to describe the main part of the variance (e.g. Littermann \& Scheinkman (1991)). Hence, if one chooses to use an $n$-factor model it is easily done by setting the remaining entries in the diagonal of $\Psi$ equal to zero. Doing a principal component analysis on the covariance matrix estimated on the full sample, we find that the first factor explains $71 \%$ of the total variance, the second $13 \%$, the third $7 \%$ and the fourth $5 \%$. That is, the first four factors account for $96 \%$ of the total variation. The results from performing the same test on sub-samples consisting of the individual years, are similar to that of the full sample regarding both the explained fractions as well as the shape of the factors. We have included the factor loadings in the Appendix A.3. For forward rates not in the sample we interpolate linearly between the factor loadings.

For the test scenarios, the initial forward rates are set to 5 percent using discrete compounding. With these assumptions we are able to compute a matrix of implied swaption volatilities from the Benchmark model using the Andersen \& Andreasen (2000) approximate swaption formula. As this matrix is input to the calibration procedure of the single-factor models, we present it in Table 1.

Table 1: Initial implied ATM swaption volatilites in the Benchmark model

\begin{tabular}{r|ccccccc}
\hline Swaption & \multicolumn{7}{|c}{ Swap Tenor } \\
Expiry & 1 & 2 & 3 & 4 & 5 & 7 & 10 \\
\hline \hline 0.5 & 16.0 & 18.4 & 18.9 & 17.9 & 16.7 & 15.1 & 13.8 \\
1.0 & 18.1 & 19.8 & 19.4 & 18.1 & 16.8 & 15.2 & 13.9 \\
2.0 & 20.5 & 20.4 & 19.2 & 17.7 & 16.5 & 15.0 & 13.8 \\
3.0 & 20.2 & 19.5 & 18.1 & 16.8 & 15.8 & 14.5 & 13.5 \\
4.0 & 19.2 & 18.3 & 17.1 & 16.0 & 15.1 & 14.0 & 13.1 \\
5.0 & 18.2 & 17.4 & 16.4 & 15.3 & 14.6 & 13.6 & 12.9 \\
7.0 & 16.7 & 16.1 & 15.2 & 14.4 & 13.8 & 13.1 & 12.5 \\
10.0 & 15.4 & 14.9 & 14.2 & 13.6 & 13.2 & 12.6 & 12.3 \\
\hline
\end{tabular}

This table contains the initial implied volatilities for ATM European swaptions computed from the Benchmark model. Swaption Expiry denotes expiry of the swaption in years and Swap Tenor denotes the maturity of the underlying swap. Thus, a 2 into 5 year swaption is a 2 year option on a 5 year swap, so the final payment from the swap is in 7 years. The payment frequency of the swaps is semi-annual.

\footnotetext{
${ }^{4}$ Thanks to Peer Roer Pedersen, Jyske Bank for delivering the yield curve information.
} 


\subsection{Finding the true Bermudan values}

In order to estimate the losses from following the exercise strategy from singlefactor models in a multi-factor world, we need to have good estimates of the true value of the Bermudan swaption. We have implemented two of the most popular methods to find tight lower bounds for American options in a simulation model. These are the non-parametric "simple approach" proposed in Andersen (2000) $(L A M)$ and the Least Squares Monte Carlo approach by Longstaff \& Schwartz (2001) (LSM). Exercise strategies from these two approaches are used as input to a Dual-Primal simulation algorithm developed in Andersen \& Broadie (2001) resulting in tight 95\%-confidence intervals for the true value given that the strategies are close to being optimal.

Without going into detail about the Primal-Dual simulation algorithms by Andersen \& Broadie (2001) (see also Haugh \& Kogan (2001)), we here shortly discuss the relation to the present value losses. The main idea in these papers is to express the primal problem of (1) as a corresponding dual problem. Andersen \& Broadie (2001) show that

$$
B S_{s, e}(t)=\inf _{\pi}\left(\pi_{t}+E_{t}^{\mathbb{Q}}\left[\max _{s \in \mathcal{T}}\left(\frac{X_{s}}{\beta_{s}}-\pi_{s}\right)\right]\right),
$$

where the infimum is taken over all $\left\{\mathcal{F}_{t}\right\}$ adapted $\mathbb{Q}$-martingales. They prove that the martingale component of the discounted Bermudan swaption price (which is supermartingale) is a solution, and they construct an approximation to this process for a given exercise strategy $\tau$. In that way the duality gap $\Delta_{t}$

$$
\Delta_{t}=E_{t}^{\mathbb{Q}}\left[\max _{s \in \mathcal{T}}\left(\frac{X_{s}}{\beta_{s}}-\pi_{s}^{\tau}\right)\right]
$$

works as a price measure of the suboptimality of the strategy $\tau$. In fact, it measures the average worst case error along all paths given that we follow a suboptimal strategy $\tau$. However, the analysis is slightly complicated by the fact that the estimate of $\Delta_{t}$ will be upward biased if the construction of $\pi_{s}^{\tau}$ requires nested simulations. Hence, we are only able to construct conservative estimates of the present value losses from following single-factor models using this method. Finally, it is important to stress that the duality gap is zero when the optimal strategy is used.

\section{$5 \quad$ Exercise strategies}

We start by listing the exercise strategies considered in this paper. First, we let a stopping time $\tau^{i}$ be defined as the first time an exercise indicator function $I^{i}(t)$ signals exercise,

$$
\tau^{i}=\inf _{t \in \mathcal{T}}\left(I^{i}(t)=1\right)
$$

where $\mathcal{T}$ is the set of possible exercise times, typically $s, \ldots, e-1$. The exercise indicator functions are in general allowed to be functions of the state variables. 
Those considered here are all based on some form of approximation of the continuation value of the Bermudan swaption $B S_{j, e}^{+}\left(t_{j}\right)$ entering the definition of the optimal strategy in (2).

\subsection{Barrier approach (LAM)}

This is the preferred strategy from Andersen (2000), though with a minor modification. Andersen (2000) uses the maximal value of the still alive core European swaptions, but Jensen \& Svenstrup (2002) show that it is much more computationally efficient to only use the first to expire, and there are no significant present value losses ${ }^{5}$.

$$
I^{L A M}\left(t_{j}\right)=\left\{\begin{array}{cc}
1 & S_{j, e}\left(t_{j}\right)^{+}>E S_{j+1, e}\left(t_{j}\right)+b\left(t_{j}\right) \\
0 & \text { else }
\end{array}\right.
$$

Here the so-called barrier function $b(\cdot)$ is a real deterministic functions $R^{+} \rightarrow$ $R^{+}$. In words the strategy is to exercise the first time the intrinsic value exceeds the sum of the European swaption maturing at the next exercise time and a constant barrier. Notice, that the barrier $b$ could be interpreted as the Bermudan premium.

\subsection{Least Square Monte Carlo approach (LSM)}

The LSM approach of Longstaff \& Schwartz (2001) consists of approximating the continuation value by a linear function of conditioning variables, $Y_{t}$, computed from a $d_{t}$-vector $Z_{t}$ of state variables

$$
B S_{i, e}^{+}\left(t_{i}\right) \approx \alpha_{t_{i}} \cdot Y_{t_{i}}, t_{i} \in \mathcal{T} .
$$

Here $Y_{t}=g_{t}\left(Z_{t}\right)$, where $g_{t}(\cdot)$ is a vector function from $R^{d_{t}} \rightarrow R^{n_{t}}$ and $\alpha_{t}$ is an $n_{t}$-parameter vector. So the exercise indicator in the LSM case is

$$
I^{L S M}\left(t_{j}\right)=\left\{\begin{array}{cc}
1 & S_{j, e}\left(t_{j}\right)^{+}>\alpha_{t_{j}} \cdot Y_{t_{j}} \\
0 & \text { else }
\end{array} .\right.
$$

Following Longstaff et al. (2001), we use the values of the core swaps as the state vector $Z_{t}$, and for the transformation $g_{t}$ we use the first three powers of the elements in $Z_{t}$ and the cross products of the values between the current swap and forward swaps up to degree three. The parameter vectors $\alpha_{t}$ are estimated using OLS as proposed in Longstaff \& Schwartz (2001) .

\footnotetext{
${ }^{5}$ We also tested the full strategy using the maximal value, and the results where virtually identical.

${ }^{6}$ In the numerical implementation we use singular value decomposition for the sake of computational stability (see e.g. Press, Flannery, Teukolsky \& Vetterling (1989)).
} 


\subsection{Single-factor approach}

Another way of estimating the continuation value is to use a more simple model, for example the single-factor models we present in the next section. For each of these single-factor models, now indicated with an asterisk, we define two exercise strategies by the following exercise indicators.

The most simple is based purely on the continuation value

$$
I^{* V}\left(t_{j}\right)=\left\{\begin{array}{cc}
1 & S_{j, e}\left(t_{j}\right)^{+}>B S_{j+1, e}^{*}\left(t_{j}\right) \\
0 & \text { else }
\end{array},\right.
$$

and simply signals exercise when the intrinsic value is larger than the continuation value in the simple model. This type of strategy we will denote with a subscript $V$ for value based.

We also test a strategy where the next to mature core European swaption observed in the market works as a form of control variate. If, for some reason, the simple model does not match this European swaption, we suggest basing the exercise decision on the Bermudan premium estimate $B P_{j+1, e}^{*}\left(t_{j}\right)=$ $B S_{j+1, e}^{*}\left(t_{j}\right)-E S_{j+1, e}^{*}\left(t_{j}\right)$ instead,

$$
I^{* P}\left(t_{j}\right)=\left\{\begin{array}{cc}
1 & S_{j, e}\left(t_{j}\right)^{+}>E S_{j+1, e}\left(t_{j}\right)+B P_{j+1, e}^{*}\left(t_{j}\right) \\
0 & \text { else }
\end{array} .\right.
$$

These strategies will be denoted with a subscript $P$ for Premium based.

\section{Single-factor interest rate models}

The single-factor models we test in this study are the Black et al. (1990) shortrate model and two single-factor forward-rate models belonging to the Heath et al. (1992) class. In particular, we make sure that these are low-dimensional Markov models such that they can be implemented in a lattice. The BDT model is one of the single-factor models tested in Longstaff et al. (2001).

\subsection{The Black, Derman \& Toy model}

The Black et al. (1990) (BDT) model is probably one of the most applied as well as one of the most severely criticized interest rate models. In the continuous time version of the model, the dynamics for the short rate $r_{t}$ can be expressed as

$$
d \ln r(t)=\left(\mu(t)+\frac{\sigma_{B D T}^{\prime}(t)}{\sigma_{B D T}(t)} \ln r(t)\right) d t+\sigma_{B D T}(t) d Z_{t} .
$$

We implement the model in a binomial tree using the forward induction algorithm described in Jamshidian (1991) to calibrate to the yield curve. One of the major drawbacks of this model is the lack of "real" mean reversion, and in order to match implied volatilities the volatility function will have to be decreasing $\sigma_{B D T}^{\prime}(t)<0$. This will effectively mean that the volatility disappears 
as time passes. We implement the BDT-tree with a volatility specification of $\sigma_{B D T}(t)=a+b \exp (-c t)$ and calibrate it to the initial prices of the European swaptions from the benchmark model using a brute-force search algorithm minimizing the relative squared price errors. The fit is not particularly satisfying with a root mean square error of $19.9 \%$. However as we are interested in testing the degree of suboptimality, this further adds strength to the test. The parameter values found are $a=13.8 \%, b=14.7 \%$ and $c=23.4 \%$. Notice that these parameters will give rise to highly non-stationary short rate dynamics, as the volatility function is very steep.

\subsection{A class of single-factor low dimensional Heath, Jarrow \& Morton models}

In this section we briefly go through a class of single-factor Heath, Jarrow \& Morton models. We consider two particular parametrizations of the model, that will allow us to value Bermudan swaptions by solving a two-dimensional partial differential equation. The model derivation is included in order to explain the calibration procedure for the second version of the model. The two versions will be denoted $R S$ and $A N$ respectively.

Heath et al. (1992) show that under the risk neutral measure $\mathbb{Q}$ the dynamics of the continuously compounded forward rates $f_{t}(T)$ must satisfy the equation

$$
f_{t}(T)=f_{0}(T)+\int_{0}^{t} \sigma(s, T) \int_{s}^{T} \sigma(s, u) d u d s+\int_{0}^{t} \sigma(s, T) d W_{s}^{\mathbb{Q}}, \quad \forall t \leq T .
$$

In this setting $W_{s}^{\mathbb{Q}}$ is a one dimensional wiener process under $\mathbb{Q}$ and $\sigma(t, T)$ is the instantaneous volatility function. In this paper we consider the class treated in e.g. Ritchken \& Sankarasubramanian (1995), where the forward volatility is of the form

$$
\sigma(t, T)=g(T) h_{t}
$$

$g(\cdot)$ is a deterministic function and $h_{t}$ is some possibly stochastic process. Substituting this into (6) we get the following

$$
f_{t}(T)=f_{0}(T)+\frac{g(T)}{g(t)}\left(x_{t}+y_{t} g(t)^{-1} \int_{t}^{T} g(s) d s\right),
$$

where $x_{t}$ and $y_{t}$ are two state variables

$$
\begin{aligned}
x_{t} & =g(t) \int_{0}^{t} h_{s}^{2} \int_{s}^{t} g(u) d u d s+g(t) \int_{0}^{t} h_{s} d W_{s}^{\mathbb{Q}} \\
& =g(t) \int_{0}^{t} h_{s}^{2} \int_{s}^{t} g(u) d u d s+\int_{0}^{t} \sigma(s, t) d W_{s}^{\mathbb{Q}}, \\
y_{t} & =g(t)^{2} \int_{0}^{t} h_{s}^{2} d s=\int_{0}^{t}\left(h_{s} g(t)\right)^{2} d s=\int_{0}^{t} \sigma(s, t)^{2} d s .
\end{aligned}
$$


By applying Leibnitz' rule the dynamics of the state variables can be seen to be

$$
d x_{t}=\left(\frac{g^{\prime}(t)}{g(t)} x_{t}+y_{t}\right) d t+g(t) h_{t} d W_{t}^{\mathbb{Q}},
$$

and

$$
d y_{t}=\left(2 \frac{g^{\prime}(t)}{g(t)} y_{t}+g(t)^{2} h_{t}^{2}\right) d t .
$$

Let $\kappa(t)=-g^{\prime}(t) / g(t)$ and let $\eta_{t}=g(t) h_{t}$ denote the volatility process of state variable $x_{t}$. If we let the $\eta_{t}$ be a function of only the state variables $x_{t}, y_{t}$ and time $t$, we have a two-dimensional Markov system. That is, let $\eta_{t}=$ $g(t) h\left(t, x_{t}, y_{y}\right)$, then

$$
\begin{aligned}
d x_{t} & =\left(-\kappa x_{t}+y_{t}\right) d t+\eta_{t} d W_{t}^{\mathbb{Q}} \\
d y_{t} & =\left(\eta_{t}^{2}-2 \kappa y_{t}\right) d t .
\end{aligned}
$$

Notice from equation (8) that the spot rate is $r_{t}=f_{t}(t)=f_{0}(t)+x_{t}$. From this it follows that the short rate evolves like

$$
d r_{t}=\frac{\partial}{\partial T} f_{0}(t) d t+d x_{t}=\left(-\kappa x_{t}+y_{t}+\frac{\partial}{\partial T} f_{0}(t)\right) d t+\eta_{t} d W_{t}^{\mathbb{Q}}
$$

A closed form solution for zero coupon bonds in this model can be proved to be

$$
\begin{aligned}
P(t, T) & =\frac{P(0, T)}{P(0, t)} \exp \left(-x_{t} \int_{t}^{T} e^{-\int_{t}^{u} \kappa(u) d u} d s-\frac{1}{2} y_{t}\left(\int_{t}^{T} e^{-\int_{t}^{u} \kappa(u) d u}\right)^{2}\right) \\
& =\frac{P(0, T)}{P(0, t)} \exp \left(-x_{t} G(t, T)-\frac{1}{2} y_{t} G(t, T)^{2}\right) .
\end{aligned}
$$

In our implementation we assume that the mean reversion function $\kappa(t)$ is constant, which implies that $g(t)=e^{-\kappa t}$ and $G(t, T)=\frac{1}{\kappa}\left[1-e^{-\kappa(T-t)}\right]$.

Finally, under the usual conditions the Feynman-Kac theorem directly implies that the value function $V\left(t, x_{t}, y_{t}\right)$ of any interest derivate solves the following 2-dimensional partial differential equation (PDE)

$$
\begin{aligned}
0 & =\frac{\partial}{\partial t} V+\left[-r+(-\kappa x+y) \frac{\partial}{\partial x}+\frac{1}{2} \eta^{2} \frac{\partial^{2}}{\partial x^{2}}+\left(\eta^{2}-2 \kappa y\right) \frac{\partial}{\partial y}\right] V \\
& =\frac{\partial}{\partial t} V+\left[-r+(-\kappa x+y) \frac{\partial}{\partial x}+\frac{1}{2} \eta^{2} \frac{\partial^{2}}{\partial x^{2}}\right] V+\left[\left(\eta^{2}-2 \kappa y\right) \frac{\partial}{\partial y}\right] V
\end{aligned}
$$

This PDE is solved using a Craig \& Sneyd split scheme described in Andreasen (2000), which is unconditionally stable and less prone to spurious oscillations, than the ordinary alternating direction implicit (ADI) scheme. Spurious oscillations are possible due to the lack of diffusion in the y-state variable. 


\subsubsection{Definition and Calibration - RS}

First, we consider a simple, but time stationary, version within this class of models. We denote it $R S$ as this version is often referred to as the Ritchken \& Sankarasubramanian (1995) model. It is contructed by setting $\eta_{t}=\sigma r_{t}^{\gamma}$ such that

$$
\sigma(t, T)=\sigma e^{-\kappa(T-t)} r_{t}^{\gamma}
$$

Unfortunately, we do not have closed form solutions for either caps or European swaptions in this version of the model, so we have chosen only to recalibrate the model initially, and as such using this model will only result in upper bounds on the losses. However, the benchmark model is also time stationary, so we expect that the volatility parameters extracted by calibrating to European swaptions are not varying much over time.

As we work with a log-normal Libor market model we let $\gamma=1$. As shown in Heath et al. (1992) the forward rate volatility function should be bounded in order to be valid. However, as there are no closed form solutions for interest rate derivatives, and as the zero coupon prices are independent of the volatility function, we are free to switch to e.g a constant volatility at some cutoff level. To represent a simple time stationary model we make an initial brute force calibration of the two parameters on a sample of ATM European swaptions with maturities of $0.5,1,2,3,4,5,7$ and 10 years written on swaps with maturities of $1,2,3,4,5,7$ and 10 years, a total of 56 swaptions. The objective in the optimization is equally weighted squared relative price errors. The optimal spot rate volatility found is $\sigma=23.1 \%$ and the mean reversion parameter $\kappa=8.3 \%$. Using only these two parameters we get a reasonable fit with a root mean square price error of $5.6 \%$. The maximal percentage error is $21 \%$ which is due to the 0.5 into 1 year swaption that the model is unable to match. In general this model is incapable of producing a volatility hump like the one observed in the benchmark model ${ }^{7}$.

\subsubsection{Definition and Calibration - AN}

This version of the model follows Andreasen (2000) and is accommodated to the pricing of Bermudan swaptions. Calibration is done individually for each of the Bermudan swaptions to the set of core European swaptions. It is a two-step procedure consisting of a separate calibration of the mean reversion level to fit the term correlation of the core swaptions, and after that a bootstrap of the variance structure of the underlying core European swaptions.

The volatility specification defining model $A N$, when pricing a $T_{e}$ no-call $T_{s}$ Bermudan swaption, is given by

$$
\eta_{t}=\alpha(t) S R_{n(t), e}(t)^{\gamma}, t \leq t_{e-1}
$$

\footnotetext{
${ }^{7}$ Rithcken \& Chuang (1999) suggest another version that allows humped volatilities but in a three state Markov system. In our experience, this hump is not particularly important for the valuation of long swaptions.
} 
where $\alpha(t)$ is a time dependent parameter vector and $S R_{n(t)}, e$ the par swap rate corresponding to the next core swap. This means that the dynamics of the par swap rate under the $k, e$-swap measure $Q_{k, e}$ (see e.g. Jamshidian (1997)) is

$$
d S R_{k, e}(t)=\frac{\partial S R_{k, e}}{\partial x}(t) \eta_{t} d W_{t}^{Q_{k, e}}, t \leq t_{k}
$$

The first step in the Andreasen (2000) calibration procedure is to approximate the term-correlation structure of the $S R_{k, n}(t)$ and $S R_{l, p}(s)$ forward swap rates by

$$
\begin{aligned}
\operatorname{corr}\left(S R_{k, n}(t), S R_{l, p}(s)\right) & \simeq \operatorname{corr}(x(t), x(s)) \\
& \simeq \sqrt{\frac{\int_{0}^{t} \exp \left(2 \int_{0}^{u} \kappa(v) d v\right) d u}{\int_{0}^{s} \exp \left(2 \int_{0}^{u} \kappa(v) d v\right) d u}} \\
& =\sqrt{\frac{1-e^{-2 \kappa t}}{1-e^{-2 \kappa s}}, s>t}
\end{aligned}
$$

and calibrate this to the term-correlation structure of the benchmark Libor market model using a constant $\kappa$ mean reversion function.

The second step utilizes the variance structure approximation

$$
\operatorname{Var}_{k, n}\left(S R_{k, n}\left(t_{k}\right)\right) \simeq \int_{0}^{t_{k}}\left(\frac{\partial S R_{k, n}}{\partial x}(u) \eta(u)\right)_{x=0, y=0}^{2} d u,
$$

which we can bootstrap to match the variance structure from the implied volatilities $\sigma_{j, e}^{2}$ of the core European swaptions for $j=s, . ., e-1$

$$
\sum_{i=s}^{j} \alpha_{i}^{2} S R_{i, n}(0)^{2 \alpha} \int_{t_{i}-1}^{t_{i}}\left(\frac{\partial S R_{j, e}}{\partial x}(u)\right)_{x=0, y=0}^{2} d u=\sigma_{j, e}^{2} S R_{j, e}(0)^{2} t_{j}
$$

Here

$$
\frac{\partial S R_{s, e}}{\partial x}(t)=S R_{s, e}(t)\left(\frac{-P\left(t, t_{s}\right) G\left(t, t_{s}\right)+P\left(t, t_{e}\right) G\left(t, t_{e}\right)}{P\left(t, t_{s}\right)-P\left(t, t_{e}\right)}+\frac{\sum_{j=s+1}^{e} \delta_{j} P\left(t, t_{j}\right) G\left(t, t_{j}\right)}{\sum_{j=s+1}^{e} \delta_{j} P\left(t, t_{j}\right)}\right) .
$$

Andreasen (2000) shows that when using this procedure the model matches the prices and skew of Bermudan swaption prices obtained in a Libor market using the LA approach.

We calibrate the term-correlation by running a one dimensional optimization over $\kappa$, where the objective is to minimize the squared absolute differences in the two term-correlation approximations. Figure 1 illustrates that we are able to match the term-correlations of the core swaptions. It should be noted that for very short swaptions the optimal mean reversion is sometimes negative, which of course is unacceptable. However, this usually happens when the termcorrelation approximation in (12) is almost insensitive to the mean reversion $\kappa$, and we therefore choose to set the mean reversion to some minimal value of $\kappa=0.5 \%$. 
Term-correlations - 10nc1

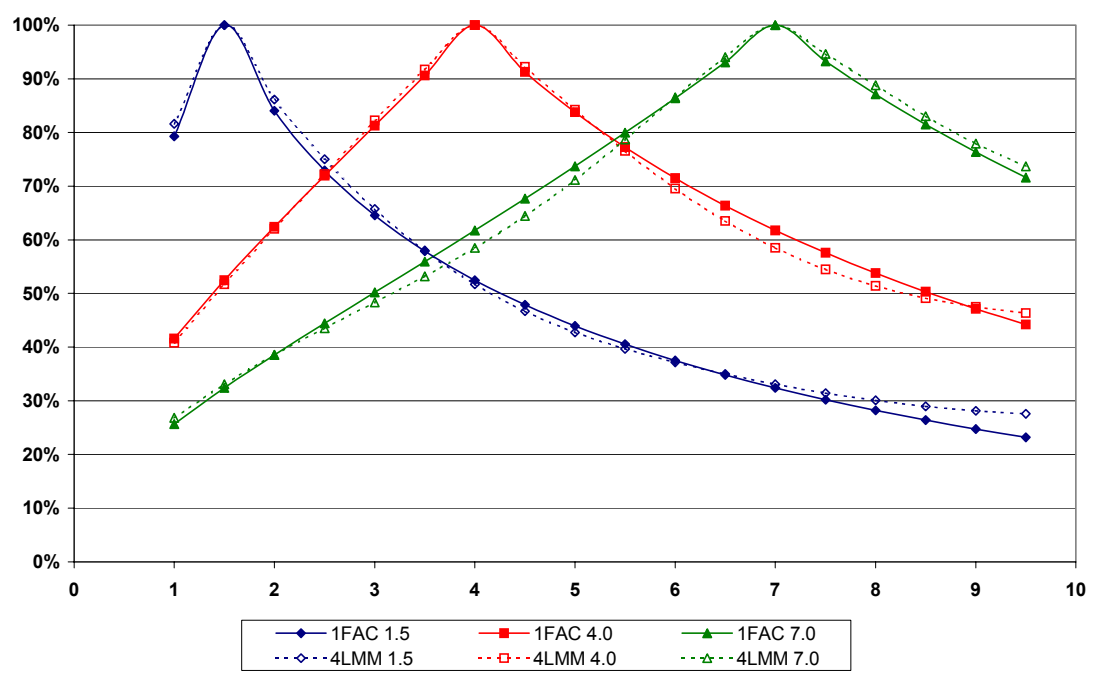

Figure 1: This figure shows an example of the calibrated term-correlations for a 10nc1 Bermudan swaption. On the first axis we have the forward times $t_{s}$ of the core swap rates. The full lines are the approximations to the term-correlations between core swap rates starting at time 1.5, 4.0 and 7.0 in the benchmark model. The dotted lines are the fitted values in the single-factor AN model.

\section{Numerical results}

\subsection{Simulation Setup}

The computation of the expectation in equation (1) is done by Monte Carlo combined with various efficiency improvement techniques. The simulation is done by simulating a log-Euler discretized version of the forward rate dynamics in (3), see for example Andersen \& Andreasen (2000).

Due to the high number of single factor valuations required in order to make the exercise decisions, we are extremely interested in keeping the number of simulation paths as low as possible. To improve the efficiency we use both antithetic variables and control variates. While implementation of antithetic variables is straightforward, the control variate setup is more involved for Bermudan swaptions. We apply the Rasmussen (2002) technique for American options which consists of sampling the value of the controls at the exercise time. Jensen \& Svenstrup (2002) demonstrate how to implement it with dividend paying assets. For Bermudan swaptions it was found that a combination of zero coupon bonds a cap control variates performed well across moneyness as well as maturities of both expiry and swap tenor. Both the LAM and the LSM approach require 
a presimulation of paths in order to determine the parameters in the exercise decision. Unless otherwise is stated we have used 25,000 antithetic paths (total of 50,000) for the presimulations. The pricing algorithms use another 250,000 antithetic paths (total of 500,000) to find the present values of the cash flow. Combined with the control variates the standard deviations of these estimates will be fairly small (around $0.1 \mathrm{bp}$ ).

\subsection{Upper bounds for the Bermudan swaption values}

In order to estimate the present value losses from following various exercise strategies, tight upper bounds for the real prices are required. Table 2 contains the lower bound estimates from both the LAM and the LMS exercise strategies as well as $95 \%$ confidence intervals for the true price. Notice that the upper bound is upward biased due to the effect of nested simulations in the dual-primal simulation algorithm. We should therefore not expect to be able to match them exactly. The duality gap $\hat{D}_{0}$ has been estimated using 1,500 antithetic paths in the outer loop and 300 antithetic paths in the inner loop, and to minimize the bias we have also used control variates (see e.g. Andersen \& Broadie (2001)).

Table 2: Price estimates and 95\% confidence intervals

\begin{tabular}{|c|c|c|c|c|c|c|c|c|}
\hline \multirow[b]{2}{*}{$t_{s}$} & \multirow[b]{2}{*}{$t_{e}$} & \multirow[b]{2}{*}{$\theta$} & \multicolumn{2}{|c|}{ Low Bound } & \multicolumn{2}{|c|}{ Duality Gap } & \multicolumn{2}{|c|}{ Upper CI 95\% } \\
\hline & & & $\hat{L}_{0}^{L S M}$ & $\hat{L}_{0}^{L A M}$ & $\hat{\Delta}_{0}^{L S M}$ & $\hat{\Delta}_{0}^{L A M}$ & $L S M$ & $L A M$ \\
\hline 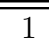 & 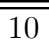 & 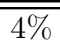 & $\overline{7767.4(0.1)}$ & $\overline{7762.7_{(0.1)}}$ & $\overline{0.5(0.0)}$ & 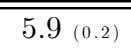 & 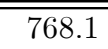 & 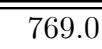 \\
\hline 1 & 10 & $5 \%$ & $394.5(0.1)$ & $391.1(0.1)$ & $0.7(0.1)$ & $3.2_{(0.2)}$ & 395.4 & 394.6 \\
\hline 1 & 10 & $6 \%$ & $205.9_{(0.1)}$ & $204.2_{(0.1)}$ & $0.2(0.0)$ & $1.7_{(0.1)}$ & 206.4 & 206.2 \\
\hline 3 & 10 & $4 \%$ & $626.4_{(0.1)}$ & $624.7_{(0.1)}$ & $0.6_{(0.0)}$ & $2.4(0.1)$ & 627.2 & 627.3 \\
\hline 3 & 10 & $5 \%$ & $355.6(0.1)$ & $354.2(0.1)$ & $0.5(0.0)$ & $1.9_{(0.1)}$ & 356.3 & 356.3 \\
\hline 3 & 10 & $6 \%$ & $196.7_{(0.1)}$ & $195.9(0.1)$ & $0.4_{(0.0)}$ & $0.7_{(0.1)}$ & 197.2 & 196.8 \\
\hline 6 & 10 & $4 \%$ & $359.7_{(0.0)}$ & $359.8(0.0)$ & $0.3_{(0.0)}$ & $0.2_{(0.0)}$ & 360.0 & 360.1 \\
\hline 6 & 10 & $5 \%$ & $222.8_{(0.0)}$ & $222.9_{(0.0)}$ & $0.2_{(0.0)}$ & $0.2_{(0.0)}$ & 223.1 & 223.2 \\
\hline 6 & 10 & $6 \%$ & $135.1_{(0.0)}$ & $135.1_{(0.0)}$ & $0.2_{(0.0)}$ & $0.2_{(0.0)}$ & 135.4 & 135.3 \\
\hline 1 & 15 & $4 \%$ & $1080.1_{(0.1)}$ & $1069.1_{(0.1)}$ & $1.4_{(0.1)}$ & $15.1(0.5)$ & 1081.7 & 1085.2 \\
\hline 1 & 15 & $5 \%$ & $578.2(0.1)$ & $570.8(0.2)$ & $1.4_{(0.1)}$ & $8.6(0.3)$ & 579.9 & 580.1 \\
\hline 1 & 15 & $6 \%$ & $318.0(0.2)$ & $315.4_{(0.2)}$ & $0.9(0.1)$ & $4.2(0.2)$ & 319.3 & 320.2 \\
\hline
\end{tabular}

$t_{s}, t_{e}$ and $\theta$ denote the lock out period, the final maturity and the coupon of the swaption. $L_{0}^{L S M}$ and $L_{0}^{L A M}$ denote the lower bound estimates from the Least Square Monte Carlo and the Andersen method, respectively. Both exercise strategies have been estimated using 25,000 AS paths in the presimulation and the price estimates are based on 250,0000 AS paths and control variates sampled using the Rasmussen (2002) method. The table also contains the estimated duality gaps as well as the upper end of the corresponding $95 \%$ confidence interval. All prices are in basis points.

The results in Table 2 demonstrate that the LAM exercise strategy generates slightly lower bounds than the LSM method. However, the duality gaps $\hat{\Delta}_{0}$ are still fairly small with a maximum of 15 basis points. The duality gaps for the 
LSM method are very small indicating that this strategy is very close to being optimal. The table contains the upper limits in a conservative $95 \%$ confidence interval for the true prices based on the upward biased duality gaps.

It is particularly interesting that the LAM method fails to pick up the last basis points for the Bermudan swaptions with long exercise periods. These findings illustrate the importance of the primal-dual algorithm of Andersen \& Broadie (2001) as it will allow us to detect exercise strategies that are far from being optimal.

Finally, these results could in principle invalidate some of the conclusions made in Andersen \& Andreasen (2001) regarding the lower prices in their twofactor model than in their single-factor model (on page 26 they report the numbers one to five basis points). To test that these differences could not be ascribed to the suboptimality of their two-factor exercise strategy, we have reconstructed their Table 6 but using the LSM exercise strategy ${ }^{8}$. The differences now decrease to about one to three basis points. It is hard to say whether differences of this size support their claim that prices are decreasing in the number of yield curve factors and not just due to different model dynamics. On the other hand it only adds strength to their claim that a single-factor model can be brought to replicate the values from a two-factor model.

\subsection{Swaption values in the single-factor models}

To provide some intuition about the fit of the single-factor models in this paper, we present Table 3, which shows the "market" prices from the Libor market model for a range of European swaptions. It also includes the prices of the Bermudan and European swaptions computed at time zero using the singlefactor models fitted to the Libor market model as described above. Finally, the Bermudan exercise premiums are included.

The European swaption prices indicate how well the single factor models fit the Libor market model. In fact, as already noted, the BDT model's fit is quite poor, in particular for OTM swaptions. The fit of the time stationary $R S$ model is reasonable, but not perfect either. The $A N$ model has a very nice fit to European swaption prices due to the calibration and especially when the lock out period is short. However, we see that the fit for the European swaptions deteriorate slightly as the lock out period $t_{s}$ increases. This is probably due to the approximations in the bootstrap calibration, where we keep the state variables fixed, and it suggests that for long European swaptions a better approximation should be used for a really tight mark-to-market ${ }^{9}$. However, for the application in this paper this should not be an issue, as we continuously recalibrate this version once the lock out period is over. Of course this is also a concern for the remaining core swaptions, but again a slack in the calibration is just another drawback for the single-factor model.

\footnotetext{
${ }^{8}$ Table is not included - but is available from the author upon request.

${ }^{9}$ For example, one could try computing the approximation keeping only $x=0$, as $y$ is locally deterministic it could be approximated using the forward curve.
} 
Table 3: Initial swaption prices from the single-factor models.

\begin{tabular}{ccc|c|ccc|ccc|ccc}
\hline & & & $L M$ & \multicolumn{3}{|c|}{$B D T$} & \multicolumn{3}{c}{$R S$} & \multicolumn{3}{c}{$A N$} \\
$t_{s}$ & $t_{e}$ & $\theta$ & $\mathrm{EU}$ & $\mathrm{B} \mathrm{S}$ & $\mathrm{EU}$ & $\mathrm{PRE}$ & $\mathrm{BS}$ & $\mathrm{EU}$ & $\mathrm{PRE}$ & $\mathrm{BS}$ & $\mathrm{EU}$ & P RE \\
\hline \hline 1 & 10 & $4 \%$ & 694.0 & 808.6 & 715.4 & 93.2 & 780.7 & 698.3 & 82.3 & 763.8 & 693.7 & 70.2 \\
1 & 10 & $5 \%$ & 193.9 & 436.7 & 255.3 & 181.4 & 418.5 & 218.4 & 200.1 & 369.6 & 191.9 & 177.7 \\
1 & 10 & $6 \%$ & 25.3 & 234.3 & 64.0 & 170.3 & 232.1 & 44.7 & 187.4 & 173.5 & 25.6 & 147.9 \\
\hline 3 & 10 & $4 \%$ & 561.4 & 644.5 & 593.0 & 51.5 & 630.3 & 566.9 & 63.4 & 615.2 & 557.2 & 58.0 \\
3 & 10 & $5 \%$ & 251.2 & 377.0 & 301.7 & 75.3 & 370.7 & 273.4 & 97.3 & 332.6 & 246.0 & 86.7 \\
3 & 10 & $6 \%$ & 93.8 & 214.6 & 138.2 & 76.4 & 218.0 & 121.8 & 96.2 & 170.4 & 90.7 & 79.7 \\
\hline 6 & 10 & $4 \%$ & 337.4 & 361.6 & 348.6 & 13.0 & 359.1 & 337.6 & 21.5 & 347.0 & 327.7 & 19.4 \\
6 & 10 & $5 \%$ & 192.2 & 224.8 & 207.6 & 17.2 & 228.3 & 200.6 & 27.7 & 204.7 & 179.3 & 25.4 \\
6 & 10 & $6 \%$ & 103.8 & 136.3 & 118.6 & 17.6 & 145.2 & 117.9 & 27.3 & 116.5 & 91.9 & 24.6 \\
\hline 1 & 15 & $4 \%$ & 960.4 & 1141.4 & 981.8 & 159.6 & 1092.1 & 960.7 & 131.5 & 1068.7 & 960.2 & 108.5 \\
1 & 15 & $5 \%$ & 248.7 & 646.6 & 325.6 & 321.1 & 602.6 & 263.2 & 339.5 & 544.6 & 246.9 & 297.7 \\
1 & 15 & $6 \%$ & 25.7 & 366.1 & 70.4 & 295.6 & 349.7 & 41.7 & 308.1 & 281.9 & 26.4 & 255.5 \\
\hline This
\end{tabular}

This table contains the prices in basis points of a set of Bermudan payer swaptions from the single factor models considered. $t_{s}, t_{e}$ and $\theta$ denote the lock out period, the final swap payment and swap coupon. LM denotes the benchmark 4-factor Libor Market model, BDT the Black, Derman and Toy, $R S$ the single-factor Rithcken \& Sankarasubramanian model and $A N$ the Andreasen model. BS and EU denote the value of the Bermudan and European swaption respectively, while PRE is the Bermudan exercise premium.

Figure 2: Initial percentage errors in the Bermudan values from the single-factor models

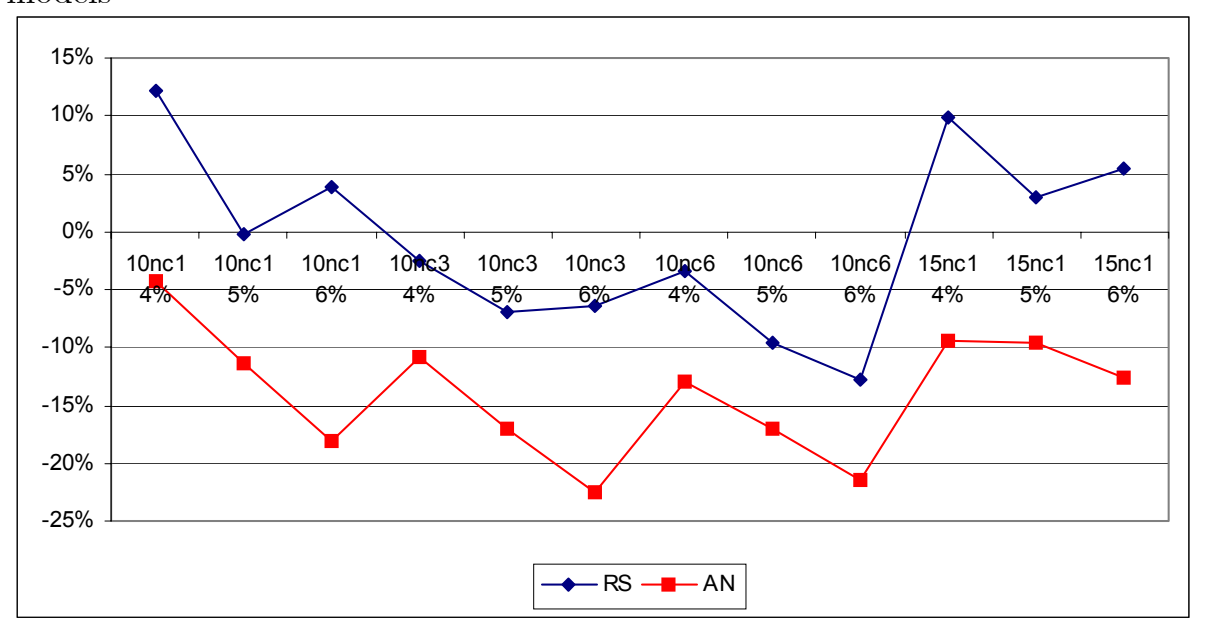

Illustration of the initial percentage errors of the single-factor forward-rate models RS and AN. Interestingly the model with the best fit to the European swaptions has larger price errors for the Bermudan swaptions. 


\subsection{Losses from following single factor exercise strategies}

Having described the benchmark model, the three-single factor models, as well as their calibration and exercise strategies, we present the results from the full simulation algorithm. Due to the computationally demanding procedure we keep the number of paths relatively low. We use 4,000 antithetic paths combined with the control variates already described.

The results presented in Table 4 confirm the findings in Andersen \& Andreasen (2001). As the $95 \%$ confidence intervals overlap with the LSM estimates it is hard to be very precise about the exact sizes of the losses (or gains) from following single-factor exercise strategies. But the bounds we are able to put on the losses are extremely small for most of the single-factor models. As expected, the Black et al. (1990) model has the worst performance, and overall the prices are lower than the LSM values. The performance of the single-factor forwardrate models, on the other hand, is comparable to that of the LSM approach. All single factor models perform better than the LAM approach. Overall, standard errors are at most of the order of a single basis point - but nothing indicates that the single-factor model's values are systematically below the LSM prices. Notice that these standard errors error actually are quite small due to the control variates. To obtain a similar precision with outcontrol variates we would roughly need 50,000 antithetic paths (100,000 total). So, even with 50,000 antithetic paths we cannot reject that the losses from following single-factor exercise strategies are zero for the best performing single-factor models.

Even if the losses cannot be distinguished from zero it is interesting to study their relative performance a little closer, as there appears to be systematic differences. In figure 3 we have computed the loss measured relative to the Bermudan premium. Notice that these estimates are much more affected by the sampling error and are to be considered with caution, but still they indicate that there are systematic differences in the relative performance of the models. In the paper of Longstaff et al. (2001) a set of common paths is used to compute these numbers. If we make the same comparison measuring the losses relative to the total contract value, instead of just the Bermudan premium, the maximal loss is only $0.03 \%$.

Figure 3 illustrates that on an overall basis the exercise strategies using the Bermudan premium, combined with the observed value of the first to expire core European swaption, is performing slightly better than the value based exercise strategies. In some way this approach works as a simple form of calibration to the first to mature European swaption.

\subsection{Conditional present value costs}

In this section we present the results in terms of the conditional present value costs as defined in Longstaff et al. (2001). The basic idea is to compare the expected losses on a common set of paths, where the single-factor strategies differ from the LSM strategy. Their argument is that we should measure the strategies where the exercise decision is a tough call, and focus more on the 
Table 4: Comparison of single-factor exercise strategies

\begin{tabular}{|c|c|c|c|c|c|c|c|c|c|}
\hline \multirow[b]{2}{*}{$t_{s}$} & \multirow[b]{2}{*}{$t_{e}$} & \multirow[b]{2}{*}{$\theta$} & \multirow{2}{*}{$L S M$} & \multicolumn{6}{|c|}{ Single - factor exercise strategies } \\
\hline & & & & $B D T_{V}$ & $B D T_{P}$ & $R S_{V}$ & $R S_{P}$ & $A N_{V}$ & $A N_{P}$ \\
\hline 1 & 10 & $4 \%$ & $\begin{array}{c}767.4 \\
(0.1)\end{array}$ & $\begin{array}{c}766.3 \\
(0.5)\end{array}$ & $\begin{array}{r}765.1 \\
(0.6)\end{array}$ & $\begin{array}{r}765.7 \\
(0.6)\end{array}$ & $\begin{array}{c}767.0 \\
(0.5)\end{array}$ & $\begin{array}{r}767.6 \\
(0.5)\end{array}$ & $\begin{array}{r}766.9 \\
(0.5)\end{array}$ \\
\hline 1 & 10 & $5 \%$ & $\begin{array}{r}394.5 \\
(0.1)\end{array}$ & $\begin{array}{r}392.4 \\
(0.7)\end{array}$ & $\begin{array}{r}394.2 \\
(0.7)\end{array}$ & $\begin{array}{r}392.6 \\
(0.8)\end{array}$ & $\begin{array}{r}393.6 \\
(0.7)\end{array}$ & $\begin{array}{r}393.7 \\
(0.7)\end{array}$ & $\begin{array}{r}395.7 \\
(0.7)\end{array}$ \\
\hline 1 & 10 & $6 \%$ & $\begin{array}{r}205.9 \\
(0.1)\end{array}$ & $\begin{array}{r}205.2 \\
(0.8)\end{array}$ & $\begin{array}{r}206.4 \\
(0.8)\end{array}$ & $\begin{array}{r}204.2 \\
(0.8)\end{array}$ & $\begin{array}{r}205.5 \\
(0.8)\end{array}$ & $\begin{array}{r}205.4 \\
(0.8)\end{array}$ & $\begin{array}{r}207.0 \\
(0.8)\end{array}$ \\
\hline 3 & 10 & $4 \%$ & $\begin{array}{c}626.4 \\
(0.1)\end{array}$ & $\begin{array}{r}625.5 \\
(0.4)\end{array}$ & $\begin{array}{c}625.9 \\
(0.4)\end{array}$ & $\begin{array}{c}625.4 \\
(0.4)\end{array}$ & $\begin{array}{r}626.2 \\
(0.4)\end{array}$ & $\begin{array}{c}626.3 \\
(0.4)\end{array}$ & $\begin{array}{r}626.0 \\
(0.4)\end{array}$ \\
\hline 3 & 10 & $5 \%$ & $\begin{array}{r}355.6 \\
(0.1)\end{array}$ & $\begin{array}{r}354.9 \\
(0.5)\end{array}$ & $\begin{array}{r}355.8 \\
(0.6)\end{array}$ & $\begin{array}{r}354.1 \\
(0.6)\end{array}$ & $\begin{array}{r}356.0 \\
(0.6)\end{array}$ & $\begin{array}{r}355.9 \\
(0.5)\end{array}$ & $\begin{array}{r}356.3 \\
(0.5)\end{array}$ \\
\hline 3 & 10 & $6 \%$ & $\begin{array}{r}196.7 \\
(0.1)\end{array}$ & $\begin{array}{r}196.3 \\
(0.6)\end{array}$ & $\begin{array}{r}196.8 \\
(0.7)\end{array}$ & $\begin{array}{r}194.7 \\
(0.7)\end{array}$ & $\begin{array}{r}196.0 \\
(0.7)\end{array}$ & $\begin{array}{r}196.5 \\
(0.7)\end{array}$ & $\begin{array}{r}196.8 \\
(0.7)\end{array}$ \\
\hline 6 & 10 & $4 \%$ & $\begin{array}{r}359.7 \\
(0.0)\end{array}$ & $\begin{array}{r}358.1 \\
(0.2)\end{array}$ & $\begin{array}{r}559.6 \\
(0.2)\end{array}$ & $\begin{array}{r}359.6 \\
(0.2)\end{array}$ & $\begin{array}{r}559.9 \\
(0.2)\end{array}$ & $\begin{array}{r}559.6 \\
(0.2)\end{array}$ & $\begin{array}{r}359.9 \\
(0.2)\end{array}$ \\
\hline 6 & 10 & $5 \%$ & $\begin{array}{r}222.8 \\
(0.0)\end{array}$ & $\begin{array}{r}221.5 \\
(0.2)\end{array}$ & $\begin{array}{r}222.3 \\
(0.2)\end{array}$ & $\begin{array}{r}222.5 \\
(0.2)\end{array}$ & $\begin{array}{r}222.8 \\
(0.2)\end{array}$ & $\begin{array}{r}222.7 \\
(0.2)\end{array}$ & $\begin{array}{r}222.8 \\
(0.2)\end{array}$ \\
\hline 6 & 10 & $6 \%$ & $\begin{array}{r}135.1 \\
(0.0)\end{array}$ & $\begin{array}{r}134.0 \\
(0.3)\end{array}$ & $\begin{array}{r}135.2 \\
(0.3)\end{array}$ & $\begin{array}{r}136.0 \\
(0.3)\end{array}$ & $\begin{array}{r}135.3 \\
(0.3)\end{array}$ & $\begin{array}{r}135.6 \\
(0.3)\end{array}$ & $\begin{array}{r}135.1 \\
(0.3)\end{array}$ \\
\hline 1 & 15 & $4 \%$ & $\begin{array}{r}1080.1 \\
(0.1)\end{array}$ & $\begin{array}{r}1079.8 \\
(0.8)\end{array}$ & $\begin{array}{r}1073.0 \\
(0.9)\end{array}$ & $\begin{array}{r}1079.4 \\
(0.9)\end{array}$ & $\begin{array}{r}1080.0 \\
(0.8)\end{array}$ & $\begin{array}{r}1078.0 \\
(0.8)\end{array}$ & $\begin{array}{r}1079.3 \\
(0.8)\end{array}$ \\
\hline 1 & 15 & $5 \%$ & $\begin{array}{r}578.2 \\
(0.1)\end{array}$ & $\begin{array}{r}575.6 \\
(1.2)\end{array}$ & $\begin{array}{r}571.3 \\
(1.2)\end{array}$ & $\begin{array}{r}577.1 \\
(1.2)\end{array}$ & $\begin{array}{r}577.3 \\
(1.2)\end{array}$ & $\begin{array}{r}578.1 \\
(1.2)\end{array}$ & $\begin{array}{r}577.4 \\
(1.2)\end{array}$ \\
\hline 1 & 15 & $6 \%$ & $\begin{array}{r}318.0 \\
(0.2)\end{array}$ & $\begin{array}{r}314.6 \\
(1.2)\end{array}$ & $\begin{array}{r}318.6 \\
(1.2)\end{array}$ & $\begin{array}{r}318.8 \\
(1.2)\end{array}$ & $\begin{array}{r}318.7 \\
(1.2)\end{array}$ & $\begin{array}{r}316.4 \\
(1.2)\end{array}$ & $\begin{array}{r}320.6 \\
(1.2)\end{array}$ \\
\hline
\end{tabular}

This table contains the results from the simulation approach. For a set of Bermudan swaptions we report the present value of the cash flows received by following the optimal strategy and exercise strategies from the described single-factor models. $t_{s}$, $t_{e}$ and $\theta$ denote the lock out time, the time of the swap maturity and coupon. LSM denotes the Least square strategy, which we have shown to be close to optimal. $B D T$, $R S$ and $A N$ denote the single-factor exercise strategies and subscripts $V$ and $P$ denote whether they are based on the Bermudan Value or Bermudan Premium plus observed European swaption. Numbers in parentheses are standard deviations. All present values are in basis points. The LSM values are based on 25,000 AS paths in the presimulation and 250,000 AS paths combined with control variates. The single-factor prices are based on 4,000 AS paths and control variates. 
Figure 3: Losses relative to the Bermudan premium
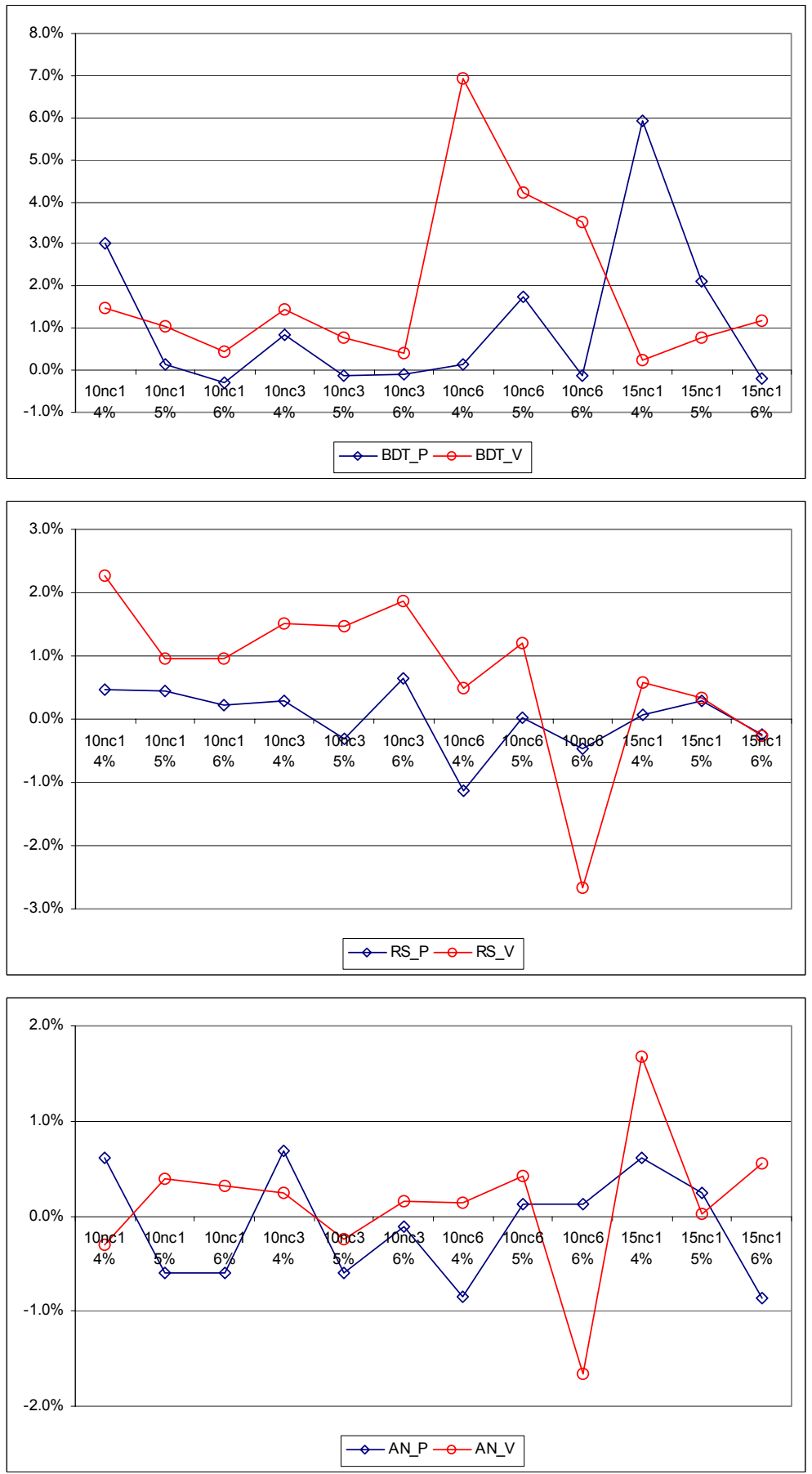

This figure plots estimates of the losses for the various single-factor exercise strategies. Percentage errors have been computed relative to the Bermudan exercise premium. 
net present values on the paths, where we make "wrong" exercise decisions. So far our results have supported the findings in Andersen \& Andreasen (2001). However, they did not investigate conditional present value costs.

Following Longstaff et al. (2001) we consider the conditional present value loss when the single-factor models signal exercise later/earlier than LSM,

$$
P V L_{\text {Late }}^{i}=E_{0}^{\mathbb{Q}}\left(\frac{X_{\tau^{L S M}}}{\beta_{\tau^{L S M}}}-\frac{X_{\tau^{i}}}{\beta_{\tau^{i}}} \mid \tau^{i}>\tau^{L S M}\right),
$$

and

$$
P V L_{\text {Early }}^{i}=E_{0}^{\mathbb{Q}}\left(\frac{X_{\tau^{L S M}}}{\beta_{\tau^{L S M}}}-\frac{X_{\tau^{i}}}{\beta_{\tau^{i}}} \mid \tau^{i}<\tau^{L S M}\right) .
$$

When we define these expectations we implicitly assume that we are not conditioning on null sets. We could easily define a stopping time $\tau$ for which one of these is undefined. Furthermore, computing these by simulation can also give rise to problems, especially when the strategies in question are very similar, as the number of observations will be low. This effect is particularly unfortunate as we are bound to keep the number of paths low. 5,000 paths were used to estimate the conditional present value losses, and the results are presented in Tables 5 (similar tables for the other models have been included in Appendix A.4). Notice that these tables include the standard deviations of the loss estimates which are not reported by Longstaff et al. (2001). Furthermore, all losses reported by Longstaff et al. (2001) are negative, which we would interpret as gains.

In Table 5 we have reported the summary statistics for the exercise strategies $\mathrm{AN}_{V}$ and LSM in the multi-factor Libor market model. Columns four and five show that the number of exercises using the single-factor model is very close to the number using the LSM from the multi-factor model. The percentage of paths where the two models signal that it is optimal to exercise at the same time, is ranging from $88.3 \%$ to $94.6 \%$, which is not particularly impressing. Despite this, present value losses associated with these differences, presented in the last columns, are overall not different from zero on a $95 \%$ level of significance. Furthermore, the signs of the losses are negative as well as positive across different swaptions. We therefore conclude that we cannot reject that the single-factor model performs just as well as the LSM strategy based on these estimates. Similar tables for the other single-factor models have been included in Appendix A.4. Not all of these perform as well as the AN models and particularly the BDT models perfom worse than the others, which is somehow what we expected given the poor dynamics and the poor initial fit to the European swaptions. On an overall basis the standard errors of the conditional loss estimates are very large.

In our opinion these results merely illustrate that this way of estimating the conditional present value loss is not very precise. The number of paths required in order to obtain an accuracy that would enable us to say somthing meaningful about present value losses using this approach, would require practically months of CPU time (at least in our implementation). 
Table 5: Comparison of the single-factor AN and four-factor Libor market model exercise strategies

\begin{tabular}{|c|c|c|c|c|c|c|c|c|c|c|c|}
\hline \multirow{2}{*}{\multicolumn{3}{|c|}{ Swaption }} & \multirow{2}{*}{\multicolumn{2}{|c|}{$\begin{array}{c}\text { Probability } \\
\text { of Exercise \% }\end{array}$}} & \multicolumn{7}{|c|}{ Single-factor Exercises } \\
\hline & & & & & \multicolumn{3}{|c|}{ Same } & \multicolumn{2}{|c|}{ Early } & \multicolumn{2}{|c|}{ Late } \\
\hline $\mathrm{t}_{s}$ & $\mathrm{t}_{e}$ & $\theta$ & $L S M$ & $A N_{V}$ & Early & time & Late & Loss & Std & Loss & Std \\
\hline 1 & 10 & $4 \%$ & 89.9 & 89.5 & 4.3 & 88.3 & 7.4 & -19 & $(14)$ & 30 & $\overline{(18)}$ \\
\hline 1 & 10 & $5 \%$ & 68.6 & 68.0 & 5.3 & 90.2 & 4.5 & 5 & $(11)$ & 3 & $(21)$ \\
\hline 1 & 10 & $6 \%$ & 46.1 & 45.6 & 3.9 & 93.8 & 2.4 & 17 & $(11)$ & 24 & $(23)$ \\
\hline 3 & 10 & $4 \%$ & 84.7 & 84.5 & 6.6 & 91.9 & 1.6 & -1 & $(8)$ & -33 & $\overline{(22)}$ \\
\hline 3 & 10 & $5 \%$ & 63.9 & 63.8 & 5.8 & 91.8 & 2.4 & -2 & $(10)$ & 13 & (18) \\
\hline 3 & 10 & $6 \%$ & 46.7 & 46.1 & 4.0 & 93.1 & 2.9 & -15 & (11) & 7 & (20) \\
\hline 6 & 10 & $4 \%$ & 78.0 & 78.1 & 9.5 & 89.0 & 1.6 & -10 & (6) & 20 & $(10)$ \\
\hline 6 & 10 & $5 \%$ & 58.4 & 58.0 & 5.5 & 92.9 & 1.7 & -11 & (6) & 5 & (9) \\
\hline 6 & 10 & $6 \%$ & 41.5 & 41.3 & 4.2 & 94.6 & 1.2 & -4 & (8) & -17 & (11) \\
\hline 1 & 15 & $4 \%$ & 89.8 & 89.9 & 5.6 & 90.0 & 4.3 & -19 & (14) & 4 & $(26)$ \\
\hline 1 & 15 & $5 \%$ & 70.5 & 70.0 & 6.9 & 88.4 & 4.7 & -23 & (13) & 12 & (24) \\
\hline 1 & 15 & $6 \%$ & 53.0 & 52.2 & 4.0 & 91.1 & 4.9 & -2 & (12) & 10 & (24) \\
\hline
\end{tabular}

This table reports summary statistics for the single-factor exercise strategy and the Least Square Monte Carlo exercise strategy in the multi-factor Libor market model. Probability of Exercise represents the total percentage of paths for which the swaption is exercised. Also the percentage of paths where the single-factor models signals exercise earlier, at the same time, or later than the multi-factor model are included. The present value losses of exercising when the single-factor model implies it is optimal at an earlier time than the four-factor model is the difference between the immediate value of exercise and the present value of cash flows generated by following the multi-factor strategy, averaged over all paths where the single-factor implies exercise earlier than. Similarly, for the present value loss of exercising when the single-factor model implies that exercise is optimal at a later time than the four-factor model. All costs are expressed in basis points. Values are based on 5,000 simulated paths of the term structure. 
We have considered the application of various efficiency improvement techniques (see e.g. Boyle, Brodie \& Glasserman (1997)), but it is not straight forward to come up with a good solution. Antithetic sampling only works well when the sampled variables are negatively correlated given two "mirror" paths. But in this case there is no reason to believe that any differences in the conditional losses for two mirror paths are highly negatively correlated. Furthermore, in order to apply the control variate technique, we are required to find some stochastic variable that is highly correlated with the loss conditional on the exercise strategies differing, and with a first moment we can compute. Such a stochastic variable is not likely to exist.

\subsection{Assessing the suboptimality using the primal-dual al- gorithm}

In order to provide more precise estimates of the conditional present value losses we compute the duality gap for the $R S_{P}$ single-factor model using the dualprimal algorithm. Testing the single-factor exercise strategies using this algorithm is a huge computational task, due to the combination of nested simulations and an exercise strategy that requires us to solve a 2-dimensional PDE. Therefore, we are forced to keep the simulation paths for the dual-primal algorithm low. Computation time is also the reason as to why we do not test the $A N$ exercise strategies even though these performed better in the previous tests.

Before commenting on the results we stress again that these estimates are in fact upward biased due to the relative low number of paths, which is also seen by comparing with the results in table 2 .

The results in Table 6 demonstrate that given the same number of paths in the primal-dual simulation algorithm, the $R S_{P}$ single-factor exercise strategy generally outperforms the multi-factor LSM approach.

Even if we use the upper end of a conservative $95 \%$ confidence interval for the losses and measure relative to the Bermudan exercise premium (which is much smaller than the total Bermudan value) we are still far from the percentage losses reported in Longstaff et al. (2001). The duality gaps have also been illustrated in Figure 4.

\section{Discussion}

Longstaff et al. (2001) stress the importance of studying single- versus multifactor models in the correct way, namely by comparing the received cash flows in the true model by using only the exercise decisions from single-factor models. The ability of a single-factor model to match the caps and European swaptions from a multi-factor model by calibration does not necessarily mean that the exercise decision for Bermudan swaptions computed within such a model is optimal.

As our results show, even for the worst performing single-factor exercise strategy, the expected losses are very limited. 
Table 6: Comparison of the duality gap for the multi-factor $L S M$ and the $R S_{P}$ single-factor exercise strategy

\begin{tabular}{ccc|c|cc|cc|cc}
\hline & & & Bermudan & \multicolumn{2}{|c|}{ Duality } & \multicolumn{2}{|c|}{ Conservative } \\
& & & Exercise & \multicolumn{2}{|c|}{ Gap } & \multicolumn{2}{c}{ Pct. Loss } & \multicolumn{2}{c}{ Pct. Loss } \\
$\mathrm{t}_{s}$ & $\mathrm{t}_{e}$ & $\theta$ & Premium & $\mathrm{D}_{0}^{L S M}$ & $\mathrm{D}_{0}^{R S_{P}}$ & LSM & $\mathrm{RS}_{P}$ & LSM & RS $_{P}$ \\
\hline \hline 1 & 10 & $4 \%$ & 73 & $0.7(0.1)$ & $0.5(0.1)$ & 0.9 & 0.6 & 1.2 & 0.9 \\
1 & 10 & $5 \%$ & 201 & $1.0(0.2)$ & $0.8(0.2)$ & 0.5 & 0.4 & 0.7 & 0.6 \\
1 & 10 & $6 \%$ & 181 & $0.4(0.1)$ & $0.4(0.1)$ & 0.2 & 0.2 & 0.3 & 0.4 \\
\hline 3 & 10 & $4 \%$ & 65 & $0.4(0.1)$ & $0.2(0.1)$ & 0.5 & 0.3 & 0.8 & 0.5 \\
3 & 10 & $5 \%$ & 104 & $0.7(0.2)$ & $0.3(0.1)$ & 0.7 & 0.3 & 1.0 & 0.5 \\
3 & 10 & $6 \%$ & 103 & $0.5(0.1)$ & $0.4(0.1)$ & 0.5 & 0.4 & 0.7 & 0.6 \\
\hline 6 & 10 & $4 \%$ & 22 & $0.6(0.1)$ & $0.1(0.0)$ & 2.8 & 0.3 & 4.0 & 0.5 \\
6 & 10 & $5 \%$ & 31 & $0.2(0.1)$ & $0.1(0.0)$ & 0.8 & 0.4 & 1.2 & 0.6 \\
6 & 10 & $6 \%$ & 31 & $0.1(0.1)$ & $0.1(0.1)$ & 0.4 & 0.4 & 0.7 & 0.7 \\
\hline 1 & 15 & $4 \%$ & 120 & $1.9(0.3)$ & $1.6(0.3)$ & 1.6 & 1.3 & 2.0 & 1.7 \\
1 & 15 & $5 \%$ & 329 & $2.0(0.3)$ & $1.7(0.4)$ & 0.6 & 0.5 & 0.8 & 0.7 \\
1 & 15 & $6 \%$ & 292 & $0.8(0.2)$ & $1.5(0.4)$ & 0.3 & 0.5 & 0.4 & 0.8 \\
\hline
\end{tabular}

This table contains the duality gaps from the multi-factor Least square Monte Carlo method (LSM) and the single-factor $R S$ exercise strategy. The Duality Gap is a measure of the expected losses from following a given strategy. The Bermudan Exercise Premium denotes the difference between the Bermudan- and the European swaption values in the multi-factor model using the LSM strategy. The Pct. Loss is the duality gap in percent of the Bermudan exercise premium. Conservative Pct. Loss denotes the duality gap plus 1.96 times the standard deviation of the duality gap relative to the Bermudan exercise premia. Duality gaps have been estimated using $200 \mathrm{AS}$ paths in the outer simulation and $25 \mathrm{AS}$ paths in the inner.

Furthermore, we applied the forward cap covering the exercise period as control variate with sampling at the exercise time. Bermduan exercise premium and the duality gap are in basis points. 
Figure 4: Duality Gaps for single-factor $R S_{P}$ and multi-factor $L S M$ exercise strategies

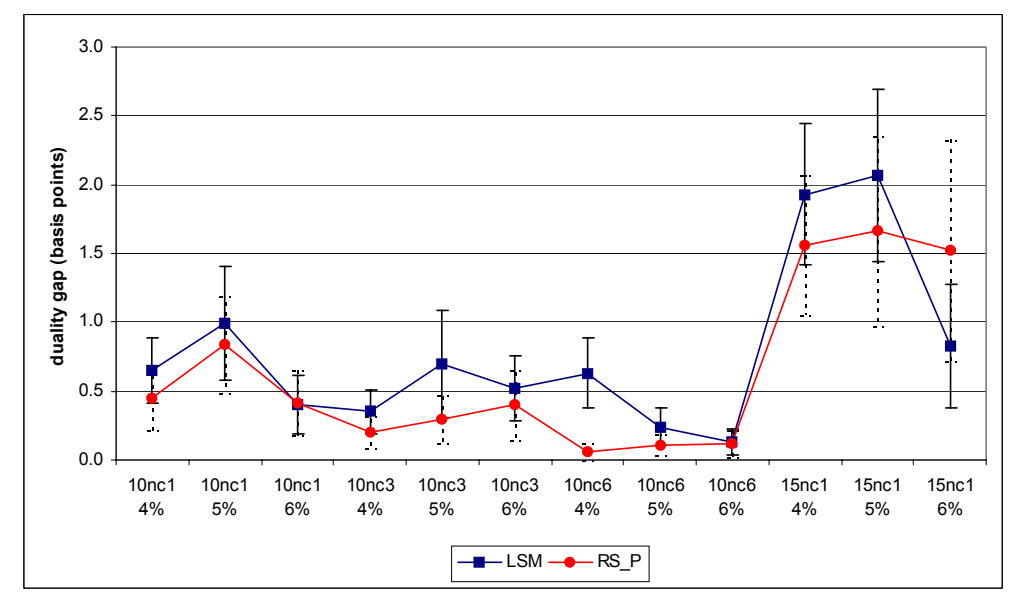

This figure demonstrates that the duality gaps from the single-factor $R S_{P}$ exercise strategy are at least as low as the multi-factor LSM, when we use the same number of paths. On the first axis we have the set of Bermudan swaptions, while the second-axis contains the duality gaps measured in basis points. 95\% confindence intervals have been included as error bars on the data series.

On top of that we have seen that the conditional present value losses based on 5,000 paths where not significantly different from zero. Even if the expected losses were positive, it seems reasonable that we also must worry about the variance of these conditional losses. If the variance is much larger than the expected loss, the holders of Bermudan swaptions could just as well get lucky. Consider, for example, a dealer, that repeatedly writes an ATM 10nc1 Bermudan swaption to a "fool", who is just following a single-factor exercise strategy in a multi-factor world. Our results show that with these odds, not even 5,000 deals are enough for the Law of Large Numbers to have locked in an almost sure profit. Hence, we claim that expected losses from following single-factor exercise strategies are also economically irrelevant.

Finally, we stress that these findings do not prove that single-factor models are able to neither hedge nor value Bermudan swaptions properly. In our opinion the ultimate test of the performance of single-factor model in the Bermudan swaptions market is their hedging performance. As already mentioned, the literature on pricing and hedging of interest rate derivatives has been growing rapidly. Hot topics of relevance in the Bermudan swaptions market, is the concept of unspanned stochastic volatility (USV) recently introduced in CollinDufresne \& Goldstein (2002), who find that straddles in the cap and floor markets cannot be hedged using bonds alone. On the other hand, Fan, Gupta \& Ritchken (2002) find that the swaptions market is well integrated with Libor swap rates and find no evidence of USV. Another research topic with relevance 
for the valuation of Bermudan swaption is the modelling of stochastic volatility and the importance of non-monotonic skews in the implied volatilities. A stochastic factor driving volatilities is likely to have a much larger effect on Bermudan swaption values than just another yield curve factor (see e.g. Jensen \& Svenstrup (2002) for some preliminary tests). Recent papers discussing the implementation and calibration of Libor market models with stochastic volatility include Andersen \& Brotherton-Ratcliffe (2001), Joshi \& Rebonato (2001).

\section{Conclusion}

We find that following exercise strategies from calibrated single-factor models in a multi-factor world does not necessarily lead to significant losses as claimed in the literature. Neither are there any indications that the conditional present value losses introduced in Longstaff et al. (2001) are important sources of risk for Bermudan swaptions. Our findings show that the losses reported in Longstaff et al. (2001) cannot be ascribed to the number of factors in the model determining the exercise strategy.

Interestingly we also find that the LSM approach outperforms the LAM approach when valuing Bermudan swaptions with long exercise periods. In fact, even the exercise strategy from the worst performing single-factor model performs better. 


\section{References}

Andersen, L. (2000), 'A simple approach to the pricing of bermudan swaptions in the multifactor LIBOR market model', Journal of Computational Finance $\mathbf{3}(2), 5-32$.

Andersen, L. \& Andreasen, J. (2000), 'Volatility skews and extensions of the libor market model', Applied Mathematical Finance 7, 1-32.

Andersen, L. \& Andreasen, J. (2001), 'Factor dependence of bermudan swaptions: Fact or fiction?', Journal of Financial Economics 62, 3-37.

Andersen, L. \& Broadie, M. (2001), 'A primal-dual simulation algorithm for pricing multi-dimensional american options', Working Paper: General Re Securities and Columbia University .

Andersen, L. \& Brotherton-Ratcliffe, R. (2001), 'Extended libor market models with stochastic volatility', Working Paper: Gen Re Securities .

Andreasen, J. (2000), 'Turbo charging the cheyette model', Working Paper, General Re Securities .

Bjerregaard Pedersen, M. (1999), 'Bermudan swaptions in the LIBOR market model', Financial Research Department, SimCorp $A / S$.

Black, F., Derman, E. \& Toy, W. (1990), 'A one-factor model of interest rates and its application to treasury bond options', Financial Analyst Journal pp. 33-39.

Boyle, P., Brodie, M. \& Glasserman, P. (1997), 'Monte carlo methods for security pricing', Journal of Economic Dynamics and Control 21, 1267-1321.

Brace, A., D. G. \& Musiela, M. (1997), 'The market model of interest rate dynamics', Mathematical Finance 7, 127-154.

Bühler, W., Uhrig-Homburg, M., Walter, U. \& Weber, T. (1999), 'An empirical comparison of forward-rate and spot-rate models for valuing interest-rate options', The Journal of Finance LIV(1), 269-305.

Carverhill, A. (1995), 'A note on the models of hull and white for pricing options on the term structure', The Journal of Fixed Income pp. 89-96.

Collin-Dufresne, P. \& Goldstein, R. S. (2002), 'Do bonds span the fixed income markets? theory and evidence for unspanned stochastic volatility.', Journal of Finance LVII(4), 1685-1730.

Driessen, J., Klaassen, P. \& Melenberg, B. (2000), 'The performance of multifactor term structure models for pricing and hedging caps and swaptions', Working Paper, Tilburg University - Center for Economic Research .

Duffie, D. (1996), Dynamic Asset Pricing, Princeton University Press. 
Fan, R., Gupta, A. \& Ritchken, P. (2002), 'Hedging in the possible presence of unspanned stochastic volatility: Evidence from swaption markets', Workin Paper Case Western Reserve University, WSOM .

Green, T. C. \& Figlewski, S. (1999), 'Market risk and model risk for a financial institution writing options', The Journal of Finance LIV(4), 1465-1499.

Gupta, A. \& Subrahmanyam, M. G. (2002), 'Pricing and hedging interest rate options: Evidence from cap-floor markets', Working Paper: Case Western Reserve University and Stern School of Business .

Haugh, M. B. \& Kogan, L. (2001), 'Pricing american options: A duality approach', Working Paper: Massachusetts Institute of Technology and University of Pennsylvania.

Heath, D., Jarrow, R. \& Morton, A. (1992), 'Bond pricing and the term structure of interest rates: A new methodology', Econometrica 60, 77-105.

Hull, J. \& Suo, W. (2001), 'A methodology for assessing model risk and its application to the implied volatility function model', Working Paper, Joseph L. Rotman School of Management, University of Toronto .

Hull, J. \& White, A. (1995), " "a note on the models of hull and white for pricing options on the term structure": Response', The Journal of Fixed Income pp. 97-102.

Hull, J. \& White, A. (1999), 'Forward rate volatilities, swap rate volatilites, and the implementation of the libor market model', Working Paper, Joseph L. Rotman School of Management, University of Toronto .

James, J. \& Webber, N. (2000), Interest Rate Modelling, John Wiley and Sons, Ltd.

Jamshidian, F. (1991), 'Forward induction and construction of yield curve diffusion models', Journal of Fixed Income 1, 62-74.

Jamshidian, F. (1997), 'Libor and swap market models and measures', Finance and Stochastics 1, 293-330.

Jensen, M. S. \& Svenstrup, M. (2002), 'Pricing bermudan swaptions in the libor market model using a primal-dual simulation algorithm', Working Paper, University of Aarhus and Aarhus School of Business .

Joshi, M. \& Rebonato, R. (2001), 'A stochastic-volatility, displaced-diffusion extension of the LIBOR market model', QUARC working paper.

Li, A., Ritchken, P. \& Sankarasubramanian, L. (1995), 'Lattice models for pricing american interest rate claims', The Journal of Finance $\mathbf{L}(2), 719-737$.

Littermann, R. \& Scheinkman, J. (1991), 'Common factors affecting bond returns', Journal of Fixed Income pp. 54-61. 
Litzenberger, R. H. (1992), 'Swaps: Plain and fanciful', Journal of Finance 47(3), 831-850.

Longstaff, F. A., Santa-Clara, P. \& Schwartz, E. S. (2001), 'Throwing away a billion dollars: The cost of suboptimal exercise strategies in the swaptions market', Journal of Financial Economics 62(62), 39-66.

Longstaff, F. A. \& Schwartz, E. S. (2001), 'Valuing american options by simulation: A simple least-squares approach', The Review of Financial Studies 14(1), 113-147.

Miltersen, K., Sandmann, K. \& Sondermann, D. (1997), 'Closed form solutions for term structure derivatives with log-normal interest rates', Journal of Finance 52, 409-430.

Press, W., Flannery, B., Teukolsky, S. \& Vetterling, W. (1989), Numerical Recipes in Pascal, Cambridge University Press.

Rasmussen, N. S. (2002), 'Efficient control variates for monte-carlo valuation of american options', Working Paper, The Aarhus School of Business .

Rebonato, R. (1996), Interest-Rate Option Models, second edn, John Wiley and Sons.

Rebonato, R. (2000), 'Accurate and optimal calibration to co-terminal european swaptions in a FRA-based BGM framework', Quarc working paper.

Rebonato, R. (n.d.a), 'Theory and practice of model risk management', working paper, Quantitative Research Centre (QUARC) of the Royal Bank of Scotland.

Rebonato, R. (n.d.b), Volatility and Correlation, John Wiley and Sons, LTD.

Ritchken, P. \& Sankarasubramanian, L. (1995), 'Volatility structures of forward rates and the dynamics of the term structure', Mathematical Finance 5, 5572.

Rithcken, P. \& Chuang, I. (1999), 'Interest rate option pricing with volatility humps', Review of Derivatives Research 3, 237-262.

Santa-Clara, P. \& Sornette, D. (2001), 'The dynamics of the forward interest rate curve with stochastic string shocks', The Review of Financial Studies 14(1), 149-185. 


\section{A Appendix}

\section{A.1 EUR Factor Loadings}

Figure 5: Factor loadings in the benchmark model

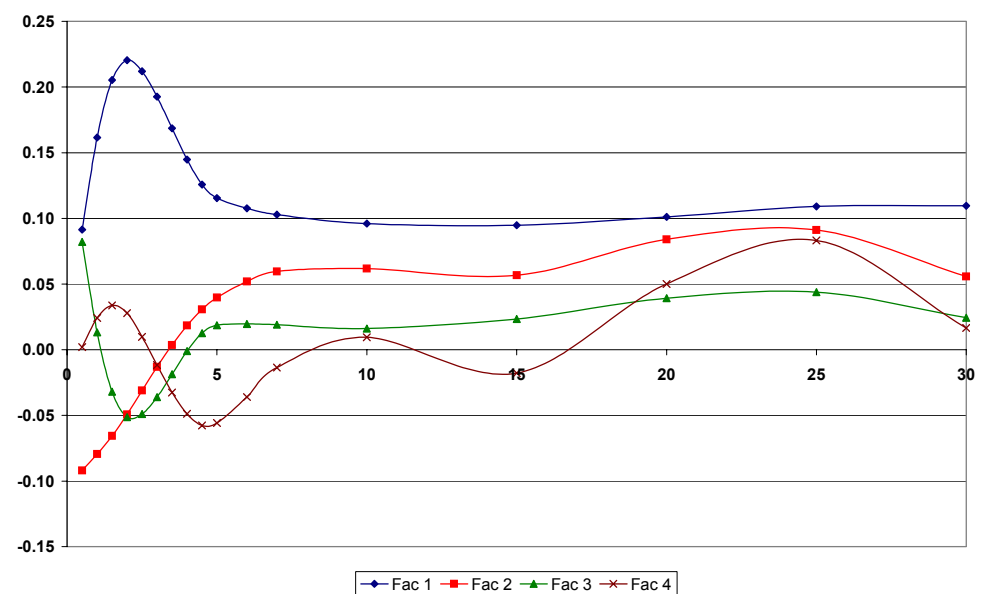

Illustration of the factor loadings for the 4-factor log-normal Libor market model used as benchmark.

Table 7: Factor loadings in the benchmark model

\begin{tabular}{c|rrrrrrrrr}
\hline Tenor & 0.50 & 1.00 & 1.50 & 2.00 & 2.50 & 3.00 & 3.50 & 4.00 & 4.50 \\
\hline 1 & 0.091 & 0.162 & 0.205 & 0.220 & 0.212 & 0.193 & 0.169 & 0.145 & 0.126 \\
2 & -0.092 & -0.079 & -0.066 & -0.049 & -0.031 & -0.013 & 0.004 & 0.018 & 0.031 \\
3 & 0.082 & 0.013 & -0.032 & -0.051 & -0.049 & -0.036 & -0.019 & -0.001 & 0.013 \\
4 & 0.002 & 0.024 & 0.034 & 0.028 & 0.010 & -0.012 & -0.033 & -0.049 & -0.058 \\
\hline Tenor & 5.00 & 6.00 & 7.00 & 10.00 & 15.00 & 20.00 & 25.00 & 30.00 & \\
\hline 1 & 0.115 & 0.108 & 0.103 & 0.096 & 0.095 & 0.101 & 0.109 & 0.110 & \\
2 & 0.040 & 0.052 & 0.060 & 0.062 & 0.057 & 0.084 & 0.091 & 0.056 & \\
3 & 0.019 & 0.020 & 0.019 & 0.016 & 0.023 & 0.039 & 0.044 & 0.024 & \\
4 & -0.056 & -0.036 & -0.014 & 0.009 & -0.018 & 0.050 & 0.083 & 0.017 & \\
\hline
\end{tabular}

Factor loadings defining the log-normal benchmark Libor market model. 


\section{A.2 Term Correlations}

The term correlation $\rho^{*}$ from time $t$ to $T$ between two variables $X_{k}$ and $X_{j}$ with instantaneous variances of $\sigma_{i}(s), i=k, j$ and instantaneous correlation $\rho_{k j}(s)$ is defined as (see e.g. Rebonato (n.d.b))

$$
\rho_{k j}^{*}(t, T)=\frac{\int_{t}^{T} \sigma_{k}(s) \sigma_{j}(s) \rho_{k j}(s) d s}{\sqrt{\int_{t}^{T} \sigma_{k}^{2}(s) d s \int_{t}^{T} \sigma_{j}^{2}(s) d s}} .
$$

\section{A.3 Vasicek Term-Correlation}

Consider the Vasicek model where the short rate dynamics is given by the SDE

$$
d r(t)=\kappa(\theta-r(t)) d t+\sigma d W(t),
$$

which have a closed form solution, $t<u$,

$$
r(u)=\theta+e^{-\kappa(u-t)}(r(t)-\theta)+\sigma \int_{t}^{u} e^{-\kappa(u-a)} d W(a) .
$$

The variance of

$$
\begin{aligned}
\operatorname{Var}(r(t)) & =\operatorname{Var}\left(\sigma \int_{t}^{u} e^{-\kappa(u-a)} d W(a)\right)=\sigma^{2} \int_{t}^{u} e^{-2 \kappa(u-a)} d W(a) \\
& =\frac{\sigma^{2}}{2 \kappa}\left(1-e^{-2 \kappa t}\right) .
\end{aligned}
$$

The time zero term-covariance is easily seen to be $t<u$,

$$
\begin{aligned}
\operatorname{Cov}(r(t), r(u)) & =\operatorname{Cov}\left(r(t), e^{-\kappa(u-t)} r(t)+\sigma \int_{t}^{u} e^{-\kappa(u-a)} d W(a)\right) \\
& =e^{-\kappa(u-t)} \operatorname{Var}(r(t)) .
\end{aligned}
$$

And from this the term-correlation function is derived

$$
\begin{aligned}
\operatorname{corr}(r(t), r(u)) & =\frac{\operatorname{Cov}(r(t), r(u))}{\sqrt{\operatorname{Var}(r(t)) \operatorname{Var}(r(u))}}=\frac{e^{-\kappa(u-t)} \operatorname{Var}(r(t))}{\sqrt{\operatorname{Var}(r(t)) \operatorname{Var}(r(u))}} \\
& =e^{-\kappa(u-t)} \sqrt{\frac{\operatorname{Var}(r(t))}{\operatorname{Var}(r(u))}}=e^{-\kappa(u-t)} \sqrt{\frac{\left(1-e^{-2 \kappa t}\right)}{\left(1-e^{-2 \kappa u}\right)}} \\
& =\sqrt{\frac{\left(1-e^{2 \kappa t}\right)}{\left(1-e^{2 \kappa u}\right)}}
\end{aligned}
$$




\section{A.4 Conditional Losses}

Table 8: Comparison of single-factor $B D T_{V}$ and four-factor Libor market model exercise strategies

\begin{tabular}{|c|c|c|c|c|c|c|c|c|c|c|c|}
\hline \multirow{2}{*}{\multicolumn{3}{|c|}{ Swaption }} & \multirow{2}{*}{\multicolumn{2}{|c|}{$\begin{array}{c}\text { Probability } \\
\text { of Exercise \% }\end{array}$}} & \multicolumn{7}{|c|}{ Single-factor Exercises } \\
\hline & & & & & \multicolumn{3}{|c|}{ Same } & \multicolumn{2}{|c|}{ Early } & \multicolumn{2}{|c|}{ Late } \\
\hline $\mathrm{t}_{s}$ & $\mathrm{t}_{e}$ & $\theta$ & LSM & $\mathrm{BDT}_{V}$ & Early & time & Late & Loss & Std & Loss & Std \\
\hline 1 & 10 & $4 \%$ & 90.3 & 88.0 & 0.1 & 76.2 & 23.7 & -12 & (41) & 15 & $\begin{array}{l}(10) \\
\end{array}$ \\
\hline 1 & 10 & $5 \%$ & 67.6 & 64.4 & 0.1 & 78.9 & 21.0 & 228 & (67) & 15 & (10) \\
\hline 1 & 10 & $6 \%$ & 46.4 & 43.7 & 0.1 & 86.5 & 13.4 & 296 & $(155)$ & 29 & $(10)$ \\
\hline 3 & 10 & $4 \%$ & 85.1 & 83.5 & 0.1 & 88.2 & 11.7 & -13 & $\overline{(39)}$ & 11 & $(9)$ \\
\hline 3 & 10 & $5 \%$ & 65.2 & 62.9 & 0.0 & 85.5 & 14.4 & 85 & $(21)$ & -3 & (9) \\
\hline 3 & 10 & $6 \%$ & 45.3 & 42.8 & 0.0 & 88.7 & 11.3 & 58 & - & 21 & (10) \\
\hline 6 & 10 & $4 \%$ & 77.0 & 75.7 & 0.3 & 94.5 & 5.2 & -61 & $(30)$ & 9 & $(5)$ \\
\hline 6 & 10 & $5 \%$ & 59.2 & 57.3 & 0.0 & 92.4 & 7.6 & 0 & - & 14 & (6) \\
\hline 6 & 10 & $6 \%$ & 40.7 & 38.5 & 0.0 & 93.1 & 6.9 & 0 & - & 16 & (7) \\
\hline 1 & 15 & $4 \%$ & 89.7 & 87.5 & 0.4 & 70.6 & 29.0 & 72 & $(45)$ & 33 & (13) \\
\hline 1 & 15 & $5 \%$ & 70.2 & 67.0 & 0.4 & 74.6 & 25.0 & -3 & $(72)$ & 2 & (12) \\
\hline 1 & 15 & $6 \%$ & 52.4 & 49.5 & 0.1 & 82.0 & 17.9 & -190 & (128) & 23 & (12) \\
\hline
\end{tabular}

This table reports summary statistics for the single-factor exercise strategy and the Least Square Monte Carlo exercise strategy in the multi-factor Libor market model. Probability of exercise represents the total percentage of paths for which the swaption is exercised. Also the percentage of paths where the single-factor models signals exercise earlier, at the same time, or later than the multi-factor model are included. The present value losses of exercising when the single-factor model implies it is optimal at an earlier time than the four-factor model is the difference between the immediate value of exercise and the present value of cash flows generated by following the multi-factor strategy, averaged over all paths where the single-factor implies exercise earlier than. Similarly, for the present value loss of exercising when the single-factor model implies that exercise is optimal at a later time than the four-factor model. All costs are expressed in basis points. Values are based on 5,000 simulated paths of the term structure. 
Table 9: Comparison of single-factor $B D T_{P}$ and four-factor Libor market model exercise strategies

\begin{tabular}{ccc|cc|rcc|rr|rr}
\hline \multicolumn{2}{c|}{ Swaption } & \multicolumn{2}{c|}{ Probability } & \multicolumn{6}{c|}{ Single-factor Exercises } & \multicolumn{2}{c}{ Late } \\
$\mathrm{t}_{s}$ & $\mathrm{t}_{e}$ & $\theta$ & LSM & BDT $_{P}$ & Early & time & Late & Loss & Std & Loss & Std \\
\hline \hline 1 & 10 & $4 \%$ & 89.4 & 88.1 & 0.5 & 82.3 & 17.3 & -3 & $(43)$ & 38 & $(12)$ \\
1 & 10 & $5 \%$ & 68.6 & 66.9 & 0.8 & 83.5 & 15.6 & 0 & $(11)$ & 31 & $(13)$ \\
1 & 10 & $6 \%$ & 47.2 & 46.1 & 0.5 & 93.0 & 6.5 & -58 & $(11)$ & 31 & $(16)$ \\
\hline 3 & 10 & $4 \%$ & 85.0 & 84.5 & 0.8 & 93.5 & 5.7 & 25 & $(8)$ & 13 & $(15)$ \\
3 & 10 & $5 \%$ & 65.9 & 65.0 & 0.6 & 92.7 & 6.6 & -53 & $(10)$ & 21 & $(14)$ \\
3 & 10 & $6 \%$ & 46.0 & 45.2 & 0.4 & 93.7 & 5.9 & -35 & $(11)$ & -25 & $(15)$ \\
\hline 6 & 10 & $4 \%$ & 77.5 & 77.0 & 0.8 & 97.2 & 2.0 & -27 & $(6)$ & 2 & $(10)$ \\
6 & 10 & $5 \%$ & 57.4 & 56.7 & 0.6 & 96.4 & 3.0 & 3 & $(6)$ & -5 & $(11)$ \\
6 & 10 & $6 \%$ & 40.5 & 40.0 & 0.9 & 97.1 & 2.1 & -21 & $(8)$ & 22 & $(12)$ \\
\hline 1 & 15 & $4 \%$ & 89.5 & 87.5 & 1.2 & 72.4 & 26.4 & -66 & $(14)$ & 20 & $(13)$ \\
1 & 15 & $5 \%$ & 68.1 & 66.6 & 0.9 & 80.3 & 18.8 & -111 & $(13)$ & 16 & $(14)$ \\
1 & 15 & $6 \%$ & 52.5 & 51.0 & 0.6 & 87.3 & 12.1 & 4 & $(12)$ & 11 & $(15)$ \\
\hline
\end{tabular}

This table reports summary statistics for the single-factor exercise strategy and the Least Square Monte Carlo exercise strategy in the multi-factor Libor market model. Probability of exercise represents the total percentage of paths for which the swaption is exercised. Also the percentage of paths where the single-factor models signals exercise earlier, at the same time, or later than the multi-factor model are included. The present value losses of exercising when the single-factor model implies it is optimal at an earlier time than the four-factor model is the difference between the immediate value of exercise and the present value of cash flows generated by following the multi-factor strategy, averaged over all paths where the single-factor implies exercise earlier than. Similarly, for the present value loss of exercising when the single-factor model implies that exercise is optimal at a later time than the four-factor model. All costs are expressed in basis points. Values are based on 5,000 simulated paths of the term structure. 
Table 10: Comparison of single-factor $R S_{P}$ and four-factor Libor market model exercise strategies

\begin{tabular}{|c|c|c|c|c|c|c|c|c|c|c|c|}
\hline \multirow{2}{*}{\multicolumn{3}{|c|}{ Swaption }} & \multirow{2}{*}{\multicolumn{2}{|c|}{$\begin{array}{l}\text { Probability } \\
\text { of Exercise \% }\end{array}$}} & \multicolumn{7}{|c|}{ Single-factor Exercises } \\
\hline & & & & & \multicolumn{3}{|c|}{ Same } & \multicolumn{2}{|c|}{ Early } & \multicolumn{2}{|c|}{ Late } \\
\hline $\mathrm{t}_{s}$ & $\mathrm{t}_{e}$ & $\theta$ & LSM & $\mathrm{RS}_{P}$ & Early & time & Late & Loss & Std & Loss & Std \\
\hline 1 & 10 & $4 \%$ & 90.3 & 990.6 & 9.8 & 87.1 & 3.1 & -8 & (14) & 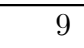 & $\overline{(22)}$ \\
\hline 1 & 10 & $5 \%$ & 67.2 & 66.5 & 6.9 & 87.1 & 6.0 & -8 & $(16)$ & 1 & $(17)$ \\
\hline 1 & 10 & $6 \%$ & 46.0 & 44.9 & 2.7 & 92.7 & 4.6 & 9 & $(20)$ & 47 & $(20)$ \\
\hline 3 & 10 & $4 \%$ & 84.5 & 84.8 & 9.8 & 88.0 & 2.3 & 4 & $(11)$ & 2 & $\overline{(16)}$ \\
\hline 3 & 10 & $5 \%$ & 65.8 & 65.4 & 5.2 & 91.7 & 3.1 & -22 & (16) & -5 & $(16)$ \\
\hline 3 & 10 & $6 \%$ & 47.3 & 46.1 & 3.0 & 92.3 & 4.7 & 20 & (18) & 13 & $(16)$ \\
\hline 6 & 10 & $4 \%$ & 77.9 & 77.5 & 6.3 & 91.6 & 2.1 & 4 & (8) & 6 & (6) \\
\hline 6 & 10 & $5 \%$ & 59.2 & 58.8 & 3.1 & 94.7 & 2.2 & -10 & (13) & 9 & (7) \\
\hline 6 & 10 & $6 \%$ & 42.2 & 41.8 & 1.8 & 96.4 & 1.7 & -9 & (17) & 3 & (9) \\
\hline 1 & 15 & $4 \%$ & 89.4 & 89.8 & 14.9 & 80.5 & 4.6 & -13 & (16) & -20 & $(25)$ \\
\hline 1 & 15 & $5 \%$ & 70.9 & 70.0 & 9.1 & 82.7 & 8.2 & -13 & (18) & 10 & $(20)$ \\
\hline 1 & 15 & $6 \%$ & 51.5 & 50.0 & 4.3 & 87.8 & 7.9 & -2 & $(22)$ & 23 & (18) \\
\hline
\end{tabular}

This table reports summary statistics for the single-factor exercise strategy and the Least Square Monte Carlo exercise strategy in the multi-factor Libor market model. Probability of exercise represents the total percentage of paths for which the swaption is exercised. Also the percentage of paths where the single-factor models signals exercise earlier, at the same time, or later than the multi-factor model are included. The present value losses of exercising when the single-factor model implies it is optimal at an earlier time than the four-factor model is the difference between the immediate value of exercise and the present value of cash flows generated by following the multi-factor strategy, averaged over all paths where the single-factor implies exercise earlier than. Similarly, for the present value loss of exercising when the single-factor model implies that exercise is optimal at a later time than the four-factor model. All costs are expressed in basis points. Values are based on 5,000 simulated paths of the term structure. 
Table 11: Comparison of single-factor $R S_{P}$ and four-factor Libor market model exercise strategies

\begin{tabular}{|c|c|c|c|c|c|c|c|c|c|c|c|}
\hline \multirow{2}{*}{\multicolumn{3}{|c|}{ Swaption }} & \multirow{2}{*}{\multicolumn{2}{|c|}{$\begin{array}{c}\text { Probability } \\
\text { of Exercise \% }\end{array}$}} & \multicolumn{7}{|c|}{ Single-factor Exercises } \\
\hline & & & & & \multicolumn{3}{|c|}{ Same } & \multicolumn{2}{|c|}{ Early } & \multicolumn{2}{|c|}{ Late } \\
\hline $\mathrm{t}_{s}$ & $\mathrm{t}_{e}$ & $\theta$ & LSM & $\mathrm{RS}_{\mathrm{V}}$ & Early & time & Late & Loss & Std & Loss & Std \\
\hline 1 & 10 & $4 \%$ & 90.2 & 89.9 & 12.5 & 83.3 & 4.3 & -7 & $\overline{(11)}$ & 14 & $\overline{(21)}$ \\
\hline 1 & 10 & $5 \%$ & 67.4 & 67.2 & 12.4 & 81.1 & 6.5 & -29 & $(11)$ & -20 & $(15)$ \\
\hline 1 & 10 & $6 \%$ & 46.0 & 44.8 & & 87.1 & 5.6 & 1 & $(11)$ & 44 & $(16)$ \\
\hline 3 & 10 & $4 \%$ & 84.4 & 85.1 & 15.7 & 82.3 & 2.1 & -1 & $(8)$ & -14 & $\overline{(18)}$ \\
\hline 3 & 10 & $5 \%$ & 64.0 & 63.7 & 11.4 & 84.7 & 3.9 & 4 & $(10)$ & 27 & $(13)$ \\
\hline 3 & 10 & $6 \%$ & 45.8 & 44.8 & 7.1 & 88.4 & 4.5 & 6 & (11) & 14 & $(15)$ \\
\hline 6 & 10 & $4 \%$ & 77.4 & 77.7 & 12.0 & 86.1 & 1.9 & -1 & (6) & -4 & (8) \\
\hline 6 & 10 & $5 \%$ & 57.8 & 57.5 & 8.5 & 89.5 & 2.0 & -1 & (6) & 5 & (8) \\
\hline 6 & 10 & $6 \%$ & 42.4 & 42.0 & 6.1 & 91.9 & 1.9 & -11 & (8) & 10 & (7) \\
\hline 1 & 15 & $4 \%$ & 89.4 & 89.3 & 15.7 & 78.6 & 5.7 & 4 & (14) & 48 & $(24)$ \\
\hline 1 & 15 & $5 \%$ & 70.1 & 68.9 & 11.7 & 78.0 & 10.3 & 13 & (13) & 29 & (18) \\
\hline 1 & 15 & $6 \%$ & 52.8 & 50.9 & 7.3 & 83.7 & 9.0 & 4 & $(12)$ & 46 & $(19)$ \\
\hline
\end{tabular}

This table reports summary statistics for the single-factor exercise strategy and the Least Square Monte Carlo exercise strategy in the multi-factor Libor market model. Probability of exercise represents the total percentage of paths for which the swaption is exercised. Also the percentage of paths where the single-factor models signals exercise earlier, at the same time, or later than the multi-factor model are included. The present value losses of exercising when the single-factor model implies it is optimal at an earlier time than the four-factor model is the difference between the immediate value of exercise and the present value of cash flows generated by following the multi-factor strategy, averaged over all paths where the single-factor implies exercise earlier than. Similarly, for the present value loss of exercising when the single-factor model implies that exercise is optimal at a later time than the four-factor model. All costs are expressed in basis points. Values are based on 5,000 simulated paths of the term structure. 
Table 12: Comparison of single-factor $A N_{P}$ and four-factor Libor market model exercise strategies

\begin{tabular}{ccc|cc|ccc|rc|rr}
\hline \multicolumn{2}{c|}{ Swaption } & \multicolumn{2}{c|}{ Probability } & \multicolumn{6}{c|}{ of Exercise $\%$} & \multicolumn{3}{c}{ Same } & \multicolumn{2}{c}{ Early } & \multicolumn{2}{c}{ Late } \\
$\mathrm{t}_{s}$ & $\mathrm{t}_{e}$ & $\theta$ & LSM & $\mathrm{AN}_{P}$ & Early & time & Late & Loss & Std & Loss & Std \\
\hline \hline 1 & 10 & $4 \%$ & 90.0 & 89.7 & 2.2 & 90.7 & 7.1 & 21 & $(23)$ & 21 & $(18)$ \\
1 & 10 & $5 \%$ & 68.5 & 68.1 & 3.0 & 91.7 & 5.3 & 9 & $(19)$ & 40 & $(19)$ \\
1 & 10 & $6 \%$ & 48.1 & 47.9 & 1.6 & 95.3 & 3.1 & -10 & $(20)$ & -1 & $(20)$ \\
\hline 3 & 10 & $4 \%$ & 84.2 & 84.4 & 3.1 & 94.0 & 2.9 & -6 & $(15)$ & 23 & $(21)$ \\
3 & 10 & $5 \%$ & 65.2 & 64.9 & 2.6 & 94.0 & 3.5 & -22 & $(18)$ & 2 & $(17)$ \\
3 & 10 & $6 \%$ & 46.7 & 46.3 & 2.0 & 95.0 & 2.9 & -1 & $(17)$ & -10 & $(22)$ \\
\hline 6 & 10 & $4 \%$ & 76.6 & 76.4 & 4.8 & 93.9 & 1.3 & -4 & $(8)$ & -2 & $(10)$ \\
6 & 10 & $5 \%$ & 58.7 & 58.6 & 2.1 & 96.9 & 1.1 & -5 & $(13)$ & 1 & $(8)$ \\
6 & 10 & $6 \%$ & 42.3 & 41.9 & 1.3 & 97.5 & 1.2 & -10 & $(14)$ & 27 & $(20)$ \\
\hline 1 & 15 & $4 \%$ & 90.1 & 90.1 & 7.3 & 87.7 & 4.9 & -6 & $(20)$ & 11 & $(26)$ \\
1 & 15 & $5 \%$ & 70.6 & 70.5 & 6.0 & 88.7 & 5.2 & -32 & $(20)$ & 4 & $(21)$ \\
1 & 15 & $6 \%$ & 51.7 & 51.7 & 2.9 & 92.6 & 4.5 & -57 & $(24)$ & -13 & $(21)$ \\
\hline
\end{tabular}

This table reports summary statistics for the single-factor exercise strategy and the Least Square Monte Carlo exercise strategy in the multi-factor Libor market model. Probability of exercise represents the total percentage of paths for which the swaption is exercised. Also the percentage of paths where the single-factor models signals exercise earlier, at the same time, or later than the multi-factor model are included. The present value losses of exercising when the single-factor model implies it is optimal at an earlier time than the four-factor model is the difference between the immediate value of exercise and the present value of cash flows generated by following the multi-factor strategy, averaged over all paths where the single-factor implies exercise earlier than. Similarly, for the present value loss of exercising when the single-factor model implies that exercise is optimal at a later time than the four-factor model. All costs are expressed in basis points. Values are based on 5,000 simulated paths of the term structure. 
Table 13: Comparison of single-factor $A N_{V}$ and four-factor Libor market model exercise strategies

\begin{tabular}{ccc|cc|ccc|rr|rr}
\hline \multicolumn{2}{c|}{ Swaption } & \multicolumn{2}{c|}{ Probability } & \multicolumn{6}{c|}{ of Exercise $\%$} & \multicolumn{3}{c}{ Same } & \multicolumn{2}{c}{ Early } & \multicolumn{2}{c}{ Late } \\
$\mathrm{t}_{s}$ & $\mathrm{t}_{e}$ & $\theta$ & LSM & AN $_{V}$ & Early & time & Late & Loss & Std & Loss & Std \\
\hline \hline 1 & 10 & $4 \%$ & 89.9 & 89.5 & 4.3 & 88.3 & 7.4 & -19 & $(14)$ & 30 & $(18)$ \\
1 & 10 & $5 \%$ & 68.6 & 68.0 & 5.3 & 90.2 & 4.5 & 5 & $(11)$ & 3 & $(21)$ \\
1 & 10 & $6 \%$ & 46.1 & 45.6 & 3.9 & 93.8 & 2.4 & 17 & $(11)$ & 24 & $(23)$ \\
\hline 3 & 10 & $4 \%$ & 84.7 & 84.5 & 6.6 & 91.9 & 1.6 & -1 & $(8)$ & -33 & $(22)$ \\
3 & 10 & $5 \%$ & 63.9 & 63.8 & 5.8 & 91.8 & 2.4 & -2 & $(10)$ & 13 & $(18)$ \\
3 & 10 & $6 \%$ & 46.7 & 46.1 & 4.0 & 93.1 & 2.9 & -15 & $(11)$ & 7 & $(20)$ \\
\hline 6 & 10 & $4 \%$ & 78.0 & 78.1 & 9.5 & 89.0 & 1.6 & -10 & $(6)$ & 20 & $(10)$ \\
6 & 10 & $5 \%$ & 58.4 & 58.0 & 5.5 & 92.9 & 1.7 & -11 & $(6)$ & 5 & $(9)$ \\
6 & 10 & $6 \%$ & 41.5 & 41.3 & 4.2 & 94.6 & 1.2 & -4 & $(8)$ & -17 & $(11)$ \\
\hline 1 & 15 & $4 \%$ & 89.8 & 89.9 & 5.6 & 90.0 & 4.3 & -19 & $(14)$ & 4 & $(26)$ \\
1 & 15 & $5 \%$ & 70.5 & 70.0 & 6.9 & 88.4 & 4.7 & -23 & $(13)$ & 12 & $(24)$ \\
1 & 15 & $6 \%$ & 53.0 & 52.2 & 4.0 & 91.1 & 4.9 & -2 & $(12)$ & 10 & $(24)$ \\
\hline
\end{tabular}

This table reports summary statistics for the single-factor exercise strategy and the Least Square Monte Carlo exercise strategy in the multi-factor Libor market model. Probability of exercise represents the total percentage of paths for which the swaption is exercised. Also the percentage of paths where the single-factor models signals exercise earlier, at the same time, or later than the multi-factor model are included. The present value losses of exercising when the single-factor model implies it is optimal at an earlier time than the four-factor model is the difference between the immediate value of exercise and the present value of cash flows generated by following the multi-factor strategy, averaged over all paths where the single-factor implies exercise earlier than. Similarly, for the present value loss of exercising when the single-factor model implies that exercise is optimal at a later time than the four-factor model. All costs are expressed in basis points. Values are based on 5,000 simulated paths of the term structure. 